\title{
Les populations de poissons dans le réservoir du Mirgenbach (1986-2006, CNPE Cattenom, France) : un modèle pour l'étude des effets d'un changement thermique global?
}

\section{Fish populations in the Mirgenbach reservoir (1986-2006, CNPE Cattenom, France) : a model to study global thermal change effects?}

\author{
G. Masson(1), S. Dembski(2), F. Staffolani(1), P. Wagner ${ }^{(1)}$, E. Valente ${ }^{(1)}$, \\ C. Maazouzi( ${ }^{(3)}$, D. Banas ${ }^{(4)}$, J.-F. Poinsaint ${ }^{(1)}$, J.-C. Pihan ${ }^{(5)}$ \\ (1) LIEBE - UMR CNRS 7146, Université Paul Verlaine, Boulevard Delestraint, 57070 Metz, France \\ (2) ONEMA, Direction de la Connaissance et de l'Information sur l'Eau, 5 square Félix Nadar, \\ 94300 Vincennes, France \\ (3) Laboratoire Écologie des Hydrosystèmes Fluviaux, UMR CNRS 5023, Université Claude Bernard \\ Lyon 1, 49 boulevard du 11 novembre 1918, 69622 Villeurbanne, France \\ (4) Université Paris-Sud, CNRS UMR 8079, Écologie, Systématique et Évolution, bâtiment 362, \\ 91405 Orsay, France \\ (5) Université Paul Verlaine, boulevard Delestraint, 57070 Metz, France \\ e-mail : masson1@univ-metz.fz
}

Résumé - Depuis 1986, le réservoir du Mirgenbach, mis en eau en 1985 reçoit les eaux échauffées issues de la centrale électronucléaire de Cattenom. Les conditions physicochimiques et la biomasse chlorophyllienne sont étudiées depuis cette date, les poissons étant échantillonnés selon un protocole standardisé depuis le couplage de la dernière tranche, en 1991. L'évolution thermique est décrite puis comparée à d'autres systèmes naturels ou échauffés. Dans le Mirgenbach, l'isolement des populations de poissons et le brassage de l'eau facilitent l'observation d'une incidence des rejets sur l'évolution du peuplement, l'abondance des populations et les traits biologiques individuels. Le seuil élevé de la température hivernale et son augmentation précoce déclenchent une reproduction et un développement individuels rapides. Le plan d'eau connaît un déclin régulier de la biomasse (chlorophylle et poissons) tandis que la contamination cuprique augmente. La diminution progressive pour plusieurs espèces, des effectifs, de la longévité, des longueurs maximales, de l'âge et de la taille à maturité sexuelle contribuent au déclin de la masse globale ou individuelle maximale. L'effet de ces contraintes s'observa d'abord sur les perches et gardons avant de s'étendre aux espèces thermophiles (brèmes communes et bordelières, perche soleil). D'après l'examen de la littérature, ces observations résulteraient de l'altération du développement sexuel et du défaut croissant en ressource trophique.

Mots clés - rejets thermiques, poisson, traits biologiques

Article published by EDF and available at http://www.hydroecologie.org or http://dx.doi.org/10.1051/hydro/2009007 


\begin{abstract}
The Mirgenbach reservoir was filled in 1985 and received since 1986 the overheated cooling waters of the electronuclear power plant of Cattenom. Physicochemical conditions and chlorophyll biomass were studied from that time. Fishes were sampled according to a standardized protocol since the coupling of the last unit in 1991. The thermal evolution was described then compared with the other natural or overheated systems. In the Mirgenbach, the isolation of fish populations with well mixed waters, facilitated the observation of incidence of the discharges on community evolution, abundance of populations and individual biological traits. The high threshold of the wintry temperature and its early rise set off the reproduction and causes a fast individual development. The reservoir knew a regular decline of the biomass (chlorophyll and fishes) whereas copper contamination increased. The progressive decrease for several species, of population size, longevity, maximal lengths, size and age of sexual maturity, contributed to the decline of the global or individual maximal mass. These constraints pressed at first on perch and roach and then on thermophilic species (bream and silver bream, pumpkinseed fish). According to literature, these constraints may be explained by a sexual developmental impairment and the increasing shortage of trophic resource.
\end{abstract}

Key words - thermal discharges, fish, biological traits

\section{INTRODUCTION}

Connaître l'amplitude et les effets du réchauffement global ou des changements climatiques revêt une importance scientifique majeure (Burroughs, 2001; Pertoldi \& Bach, 2007). Dans les écosystèmes aquatiques, l'instabilité thermique peut avoir des conséquences sévères pour les organismes dépourvus de mécanismes physiologiques régulant la température corporelle (Wood \& McDonald, 1997). Les réponses physiologiques du poisson exposé à des conditions thermiques particulières ont été étudiées soit pour optimiser et contrôler la production dans un contexte aquacole, soit pour éprouver les capacités de résistance individuelle à un stress (Jobling, 1994; McCarthy \& Houlihan, 1997 ; Beitinger et al., 2000). Ainsi, dans le cas d'un choc thermique, les conditions vitales ou optimales pour une espèce et un stade particuliers s'apparentent à un prisme dont les contours intègrent la température d'adaptation, la température et la durée d'exposition.
Cependant, il existe un nombre limité d'espèces auquel ce modèle soit appliqué (Beitinger et al., 2000). En outre, l'exposition d'individus à un accroissement thermique ne peut se résumer en termes de facteur de risques immédiats.

La température détermine l'activité anabolique, elle conditionne la prise alimentaire, la synthèse protéique, la croissance, l'ontogenèse et le développement sexuel (Wood \& McDonald, 1997). Les dépenses cataboliques étant amplifiées par une température croissante, la dépense d'énergie non compensée par une prise alimentaire adéquate mobilise les réserves énergétiques et provoque un ralentissement de croissance, compromet le développement sexuel ou entraîne l'épuisement des réserves et la disparition de l'animal si le bilan énergétique (énergie entrante énergie dépensée) reste déficitaire (Jobling, 1994, 1997).

Ces tests menés en conditions contrôlées et homogènes concernent des organismes isolés, conditionnés 
et confinés pendant une durée courte devant la durée du cycle vital. L'extrapolation de ces résultats de la biologie des organismes à la biologie des populations est délicate, de même que l'identification des effets d'une dérive thermique pour une population vivant en milieu ouvert. L'aire biogéographique devient alors un modèle pour déceler des changements de traits biologiques (croissance, âge de maturité, longévité) entre populations vivant à des latitudes et des longitudes voire des altitudes différentes (Bruslé \& Quignard, 2004). Par ailleurs, certaines études halieutiques fournissent des traits de populations variant par leur localisation et leur niveau d'exploitation (Reynolds et al., 2001). D'autres populations apportent une évolution des traits sous l'influence conjuguée de pression d'exploitation par pêche et de changement climatique à l'échelle de quelques décennies (Glantz, 1992 ; Krovin \& Rodionov, 1992; Brander, 1996; Law, 2001). Le comportement des populations en limite de répartition biogéographique constitue un indicateur d'exposition dans des territoires où la température participe au contrôle de l'expansion spécifique. Dans le cas du bar, Dicentrarchus labrax, les effets du changement climatique se font ressentir à la fois par un gain de croissance dans l'aire de répartition septentrionale, par l'extension de ce territoire et une réduction des conditions de recrutement dans la partie méridionale (Bruslé \& Quignard, 2004). Dans le cas de la perche soleil Lepomis gibbosus, Copp et al. (2004) associent les potentialités de prolifération aux conditions de croissance qui sont modulées par les gradients thermiques au sein de l'aire biogéographique.
Dans les eaux courantes, si la température moyenne du mois le plus chaud entre dans le calcul d'indices pour prédire la qualité des peuplements dans un cours d'eau (Verneaux, 1968; Oberdorff et al., 2002 ; CEN \& AFNOR, 2004), la faiblesse relative des accroissements thermiques permet d'évaluer les effets sur les communautés (Daufresne et al., 2003) mais il est plus difficile d'étudier à moyen terme, leurs conséquences sur les traits biologiques individuels, en raison du glissement graduel des peuplements de l'aval vers l'amont. En lac, les effets hydrologiques et biologiques de l'échauffement sont plus manifestes. Gillet \& Quétin (2006), en s'appuyant sur une chronique de plus de 15 ans décrivent l'incidence d'un accroissement thermique sur la reproduction du gardon Rutilus rutilus. Cependant, l'existence de gradients thermiques, notamment verticaux complique l'évaluation du bilan d'exposition individuelle (nombre de degrés jours vécus par écophase).

Accroître de manière significative, en milieu ouvert, la température d'exposition des populations de poissons, pendant plusieurs générations tout en limitant les écarts thermiques instantanés aux échelles spatiales, sans modifier les autres conditions abiotiques paraît illusoire puisque cette conception écarte les lacs soumis à la présence de thermocline et d'oxycline saisonnières ainsi que les eaux courantes où l'accroissement estival de température s'accompagne par exemple de variations de teneur en oxygène, d'écoulement, de hauteur d'eau, de surface immergée et de continuité longitudinale ou de connectivité latérale. 
Certains sites échauffés réunissent pourtant ces conditions lorsque les rejets industriels alimentent un hydrosystème ouvert mais à lent renouvellement : Bassin Biotest (Forsmark, Suède; Luksiene et al., 2000), baie Hamnefjarden (Oskarshamn, Suède; Andersson et al., 1990), Lac Druksiai (Ignalina, Lituanie; Balkuviené \& Pernaraviciuté, 1994; Bertonas, 2002), Meuse (Tihange, Belgique; Mattheeuws et al., 1981). Les travaux menés concernent la croissance, la maturation sexuelle du poisson et ses dysfonctionnements ainsi que leurs conséquences pour l'abondance des populations et leur structure (Karas \& Neuman, 1981; Luksiene \& Sandstrom, 1994; Van der Kraak \& Pankhurst, 1997). Il existe un nombre limité d'études ou de sites permettant une telle description des effets à moyen terme de l'échauffement des eaux sur la biologie des populations (Wood \& McDonald, 1997). Dans les sites ouverts, les effets pluriannuels de l'exposition des poissons aux rejets thermiques dépendent cependant de l'éloignement des populations et de leur mobilité; ces écarts d'exposition peuvent induire des gradients de caractère (Gajdusek et al., 1987).

Le réservoir du Mirgenbach (Cattenom, France) connaît un renouvellement quasi constant; sa température est relativement homogène dans l'espace (Vein et al., 1990). II revêt une originalité particulière, puisque les populations de poissons peuvent être considérées comme confinées depuis la colonisation du réservoir par pompage en Moselle, en 1985 ou les déversements volontaires ultérieurs (Dembski, 2005). La maturation sexuelle des perches Perca fluviatilis et des perches soleil y est précoce (Flesch, 1994 ; Dembski, 2005 ; Dembski et al., 2006 ; Valente, 2008). L'abondance des perches fluviatiles a diminué lors du couplage progressif des tranches électronucléaires au réservoir (Flesch et al., 1994) de même que les indicateurs de biomasse pour le peuplement de poissons dans le domaine pélagique entre 1991 et 2003 (Dembski, 2005 ; Dembski et al., 2007). À notre connaissance, aucun autre site instrumenté ne réunit les possibilités d'examiner les effets à moyen terme d'un échauffement (de plusieurs degrés Celsius) sur le poisson à l'échelle de la communauté tout en considérant l'occupation spatiale et des éléments de la dynamique de population des espèces. L'objectif de cette synthèse sera donc à la fois de caractériser les traits biologiques du poisson ainsi que la composition de l'ichthyocénose vivant depuis plusieurs générations dans le Mirgenbach (1986-2006) et d'analyser les limites d'utilisation de ce modèle pour apprécier les risques induits par un accroissement thermique.

\section{CARACTÉRISATION DU MODĖLE MIRGENBACH}

Le site électronucléaire de Cattenom, localisé dans le département de la Moselle sur le ban communal de Cattenom se situe à $8 \mathrm{~km}$ au NordEst de Thionville et à proximité du Luxembourg et de l'Allemagne. En 1985, dans le cadre de l'aménagement qui a accompagné l'implantation de la centrale électronucléaire, le réservoir artificiel du Mirgenbach est créé par barrage du ruisseau du Mirgenbach, affluent senestre au premier degré de la rivière Moselle 


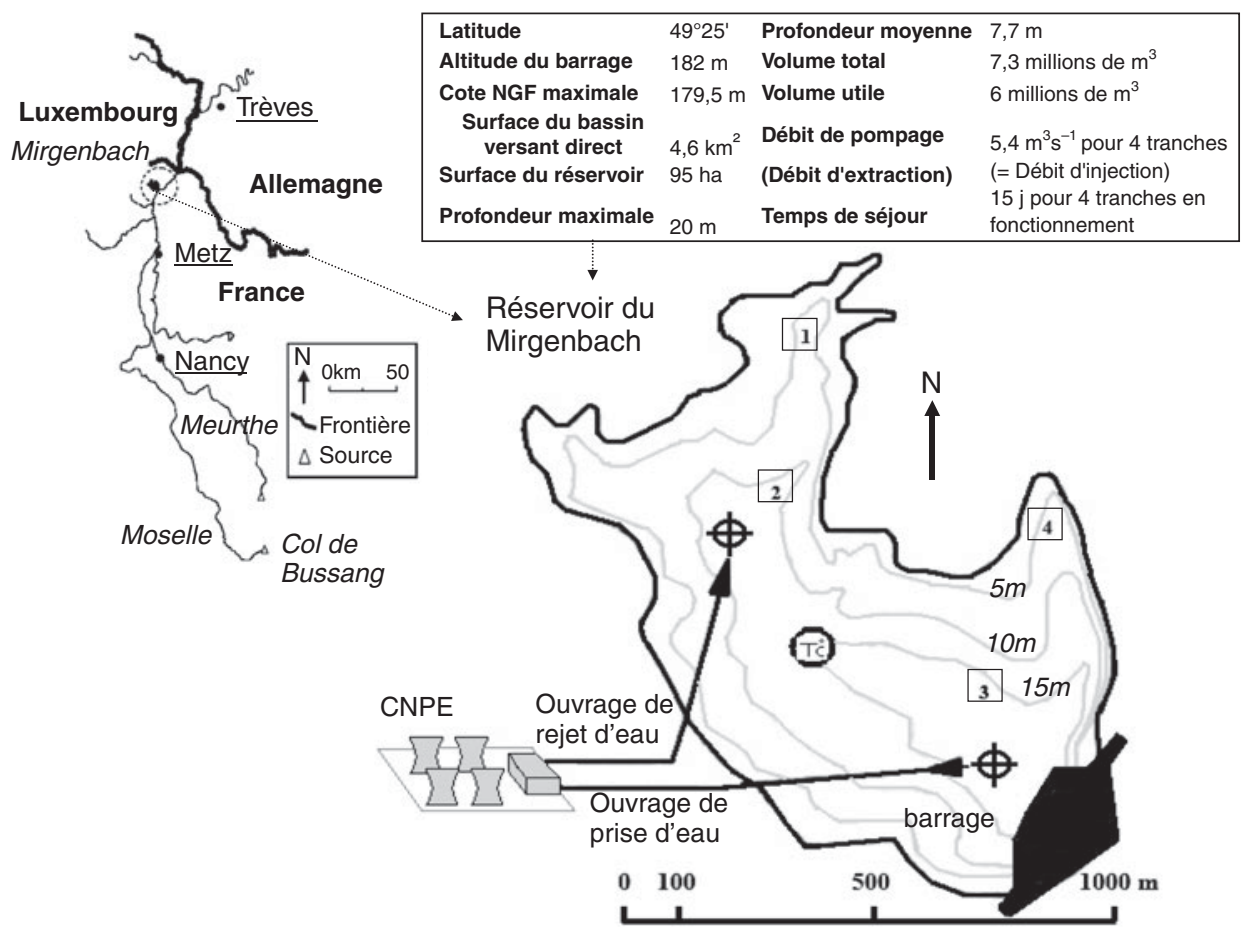

Fig. 1. Réservoir du Mirgenbach. Localisation des stations d'échantillonnage. 1, 2, 3, $4:$ station de pêche aux filets maillants verticaux. $\mathrm{T}^{\circ} \mathrm{C}$ : température et chlorophylle.

Fig. 1. Mirgenbach reservoir. Field stations. 1, 2, 3 and 4: fish sampling stations (vertical gill nets). $\mathrm{T}^{\circ} \mathrm{C}$ : temperature and chlorophyll.

(Fig. 1). La mise en eau de la retenue par pompage des eaux de la rivière Moselle date d'avril à octobre 1985; le couplage de la première tranche énergétique a eu lieu en octobre 1986, de la deuxième en août 1987 , des troisième et quatrième respectivement en février 1990 et mai 1991. L'emprise totale du site de la centrale et du plan d'eau représente 415 hectares, le réservoir occupant à lui seul 95 hectares pour un volume utile de $7,3 \times 10^{6} \mathrm{~m}^{3}$, une profondeur moyenne de 7,68 mètres avec un maximum de 20 mètres et un temps de séjour moyen de 15 jours avec un débit d'injection d'environ $5 \mathrm{~m}^{3} / \mathrm{s}$ constitué en majorité des eaux de purge des aéroréfrigérants. La retenue non vidangeable constitue en priorité une réserve pour l'alimentation des circuits de sauvegarde mais joue également le rôle de tampon thermique puisqu'au cours de leur séjour dans le plan d'eau, les eaux issues du circuit tertiaire de refroidissement subissent un échange air/eau sur une superficie de 95 ha, avant leur rejet en Moselle.

De par sa vocation, la retenue présente des caractéristiques physicochimiques et hydrobiologiques originales (Pihan, 1986 à 2005, Vein et al., 
1990 ; Dubost, 1996; Vinot \& Pihan, 2005 ; Dembski et al., 2007; Maazouzi et al., 2008a, 2008b). L'été, les aéroréfrigérants contribuent à un meilleur refroidissement des eaux alimentant le Mirgenbach qu'en hiver. La température n'a jamais excédé $30^{\circ} \mathrm{C}$ en été, même lors de la canicule 2003, alors qu'en hiver, elle dépasse $8^{\circ} \mathrm{C}$ (Fig. 2). La masse d'eau s'échauffe dès le début du mois de mars, la température dépassant souvent les $10^{\circ} \mathrm{C}$. Un régime de température caractérisé par un automne prolongé, un hiver doux et un printemps précoce est caractéristique des rejets thermiques issus d'une centrale énergétique (Luksiene et al., 2000). En Suède, dans le bassin Biotest (Forsmark) et la baie Hamnefjarden (Oskarshamn), la température suit une évolution saisonnière voisine des sites témoins : les écarts thermiques annuels entre ces sites industriels et leurs témoins varient respectivement de 5 à $10^{\circ} \mathrm{C}$ et de moins de $3^{\circ} \mathrm{C}$ à $10^{\circ} \mathrm{C}$. Dans le lac Druksiai (Ignalina, Lituanie), la même amplitude apparaît (Luksiene et al., 2000). Ces systèmes fournissent des modèles amplifiés des accroissements thermiques naturels relevés dans le Leman $\left(1^{\circ} \mathrm{C}\right.$ entre 1983 et 2000, Gillet \& Quétin, 2006) ou annoncés, par exemple en Angleterre dans le Lake District : il est pronostiqué une progression d'ici 2050 , de $1,08^{\circ} \mathrm{C}$ l'hiver, dans le lac le moins profond et de $2,18^{\circ} \mathrm{C}$, l'été dans le lac dont la thermocline est la moins profonde (George et al., 2007).

La qualité physicochimique de la retenue du Mirgenbach est tributaire de celle des eaux de la rivière Moselle à la prise d'eau, dont une caractéristique principale est une conductivité et une dureté élevées depuis la confluence de la Meurthe recevant les effluents très minéralisés des salines (rejet

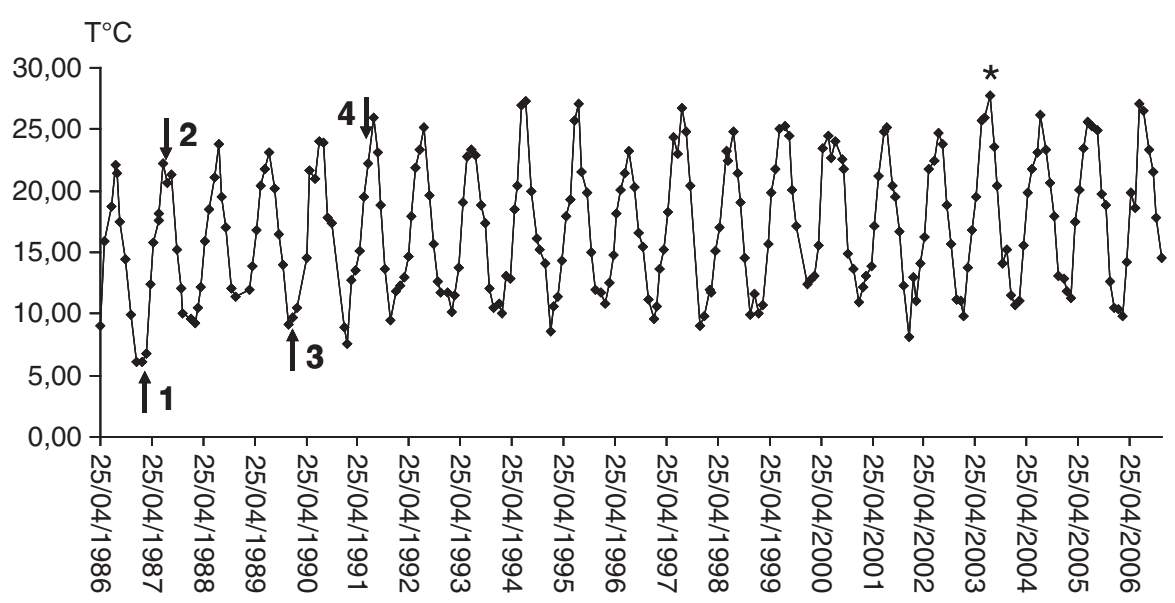

Fig. 2. Évolution de la température dans le réservoir du Mirgenbach. 1 à 4: mise en fonctionnement successive des tranches électronucléaires. * : canicule 2003.

Fig. 2. Variation of temperature in Mirgenbach reservoir. 1 to 4 : start of electronuclear units. *: canicule 2003. 
de $\mathrm{NaCl}$ ) et des soudières (rejet de $\mathrm{CaCl}_{2}$ ). La Moselle se caractérise au niveau national par une conductivité $\left(1680 \mu \mathrm{S} . \mathrm{cm}^{-1}\right)$ et une dureté $(566 \mathrm{mg}$ de carbonate de calcium par litre) exceptionnellement élevées. Le fonctionnement de la centrale a plusieurs conséquences sur cette qualité avec un échauffement des eaux, une augmentation de la concentration des sels dissous au niveau des aéroréfrigérants (facteur $\times 1,7$ ). Par ailleurs le transit continu du débit injecté par la centrale dans le réservoir explique l'absence de marnage (hors période de contrôle du barrage) et crée un brassage dans la retenue, favorable à une oxygénation du fond et à la non constitution d'une thermocline ou d'une chimiocline. Cette relative homogénéité verticale s'étend à l'ensemble de la masse d'eau; les gradients thermiques entre la rive et le large sont modérés (Dembski, 2005). La stabilité de la cote établie à 179,7 m NGF, en condition de fonctionnement normal, permet l'installation d'une végétation macrophytique pérenne.

La surverse de la retenue dans le ruisseau du Mirgenbach n'existe que lorsque de fortes précipitations alimentent le bassin versant direct du ruisseau. Cet écoulement élève alors le niveau du réservoir qui reçoit surtout les eaux originaires et dérivées depuis la Moselle (bassin versant indirect). Le débit restitué en Moselle à l'ouvrage de rejet $\left(5 \mathrm{~m}^{3} . \mathrm{s}^{-1}\right.$ environ) et en provenance de la retenue dilue les rejets radioactifs liquides conformément au cahier des charges et doit correspondre à une qualité définie dans l'arrêté d'exploitation. L'écosystème récepteur, la rivière Moselle, doit maintenir une qualité définie après mélange des eaux.
L'étude d'impact (Université de Metz, UER écologie \& Institut européen d'écologie, 1977) avant installation avait principalement attiré l'attention sur les risques prévisibles de déséquilibre dans la retenue à savoir : eutrophisation critique pouvant atteindre l'hypereutrophisation (concentration des nitrates et phosphates) avec stratification, désoxygénation et fleurs d'eau. Postérieurement au remplissage par les eaux de la Moselle, le Mirgenbach a évolué d'un niveau eutrophe vers un niveau oligotrophe par épuisement des nutriments. Lors de la mise en fonctionnement de la première tranche (automne 1986), le Mirgenbach est devenu rapidement eutrophe en raison du renouvellement et de la circulation de la masse d'eau. Toutefois, depuis 1991 le rapport N/P moyen annuel n'est globalement qu'en très légère augmentation, laissant supposer une relative stabilité trophique du système (Dembski et al., 2007). Si la stratification peut s'installer seulement en cas d'arrêt des débits de purge vers la retenue (Dubost, 1996), l'eutrophisation qui s'est manifestée nettement au début des années 1990, au terme du raccordement de la quatrième tranche (1992) s'exprime moins depuis les années 2000. Selon les auteurs, cette évolution provient de facteurs propres au système (brassage permanant, profondeur de mélange, turbidité, accumulation de cuivre) ou externes au système (évolution de la qualité et de la nature des apports de la Moselle, apports de cuivre issus de la centrale). La production primaire décline alors que la concentration du cuivre dans l'eau et les sédiments a progressé avec les années (Fig. 3; Mersch et al., 1993; Vinot, 


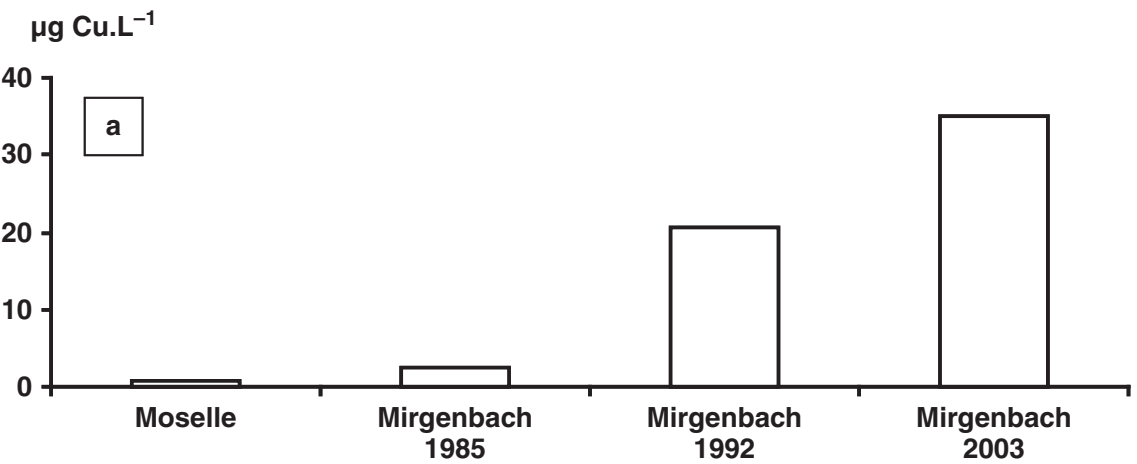

mg Cu. $\mathrm{kg}^{-1}$

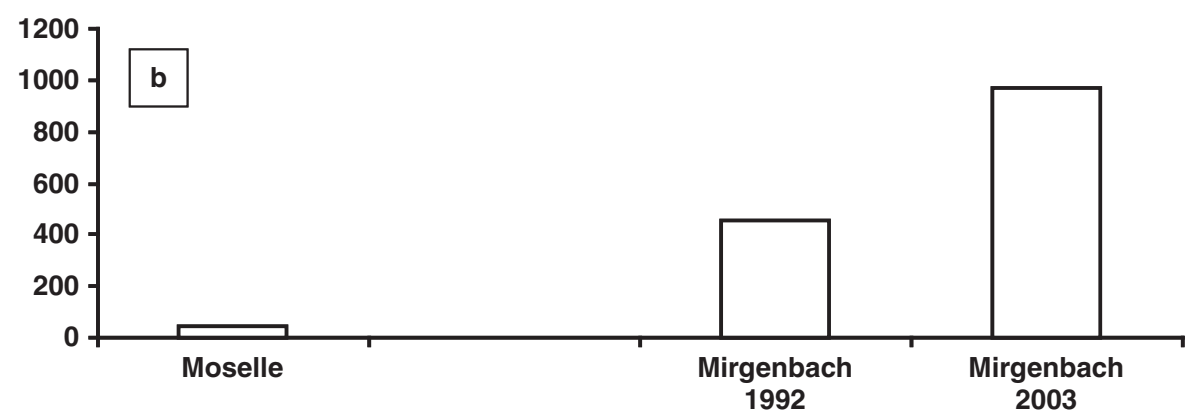

Fig. 3. Évolution de la concentration en cuivre (Cu) (a) dans l'eau brute $\left(\mu \mathrm{gCu} . \mathrm{L}^{-1}\right)$ et (b) dans le sédiment (mgCu. $\mathrm{kg}^{-1}$ de matières sèches).

Fig. 3. Variation of Cupric (Cu) concentration: (a) in water $\left(\mu \mathrm{gCu} . \mathrm{L}^{-1}\right)$ and (b) in sediment $\left(\mathrm{mgCu} \mathrm{kg}^{-1}\right.$ of dry matter).

2004 ; Vinot \& Pihan, 2005) et que dans le même temps, la transparence tend à décliner (Gosse, 2008). Or, le couple turbidité - profondeur de mélange est défavorable à la production (Gosse, 2008). La libération conjointe de cuivre et de zinc provient de la corrosion des tubes en laiton des échangeurs de chaleur entre les circuits secondaire et tertiaire. L'aptitude à la biologie est mauvaise si l'on considère la concentration totale en cuivre dans l'eau $\left(40 \leq \mathrm{Cu} \leq 110 \mu \mathrm{gCu}\right.$. $^{-1}$ pour une dureté $\left.>200 \mathrm{mgCaCO}_{3} \cdot \mathrm{L}^{-1}\right)$. Le cuivre exerce un pouvoir algicide variable selon les familles d'algues présentes et même selon le genre ou l'espèce puisque son écotoxicité varie dans une large gamme de concentration. Ainsi, selon Winner \& Owen (1991), la richesse spécifique du phytoplancton diminue entre 20 et $40 \mu \mathrm{gCu} . \mathrm{L}^{-1}$ ce qui pourrait expliquer la diminution de la concentration moyenne annuelle en chlorophylle totale constatée depuis 1998 (Fig. 4). Cependant, de 1992 à 2003, Gosse (2008) a relevé que la baisse des 


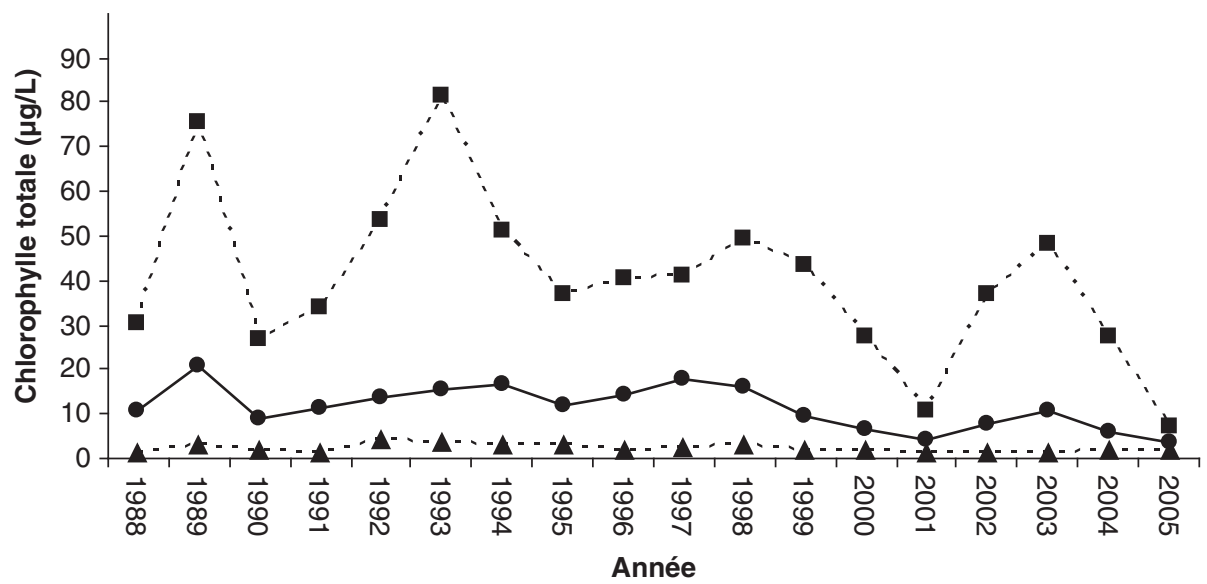

Fig. 4. Évolution de la concentration en chlorophylle totale dans la retenue du Mirgenbach (valeurs mensuelles maximales, moyennes et minimales).

Fig. 4. Variation of total chlorophyll concentration in the Mirgenbach reservoir (maximum, average and minimum of monthly data).

teneurs annuelles de chlorophylle a dans le réservoir suit globalement celle observée dans la Moselle qui ensemence le réservoir en phytoplancton. Les niveaux de consommateurs présents dans l'écosystème (zooplancton, invertébrés benthiques ou de pleine eau, poissons) subissent l'action directe du cuivre en fonction de leur sensibilité vis-à-vis de ce micropolluant (Vinot 2004 ; Vinot \& Pihan, 2005), de l'échauffement des eaux et indirectement du fait de la sélection des espèces planctoniques limitant le choix alimentaire pouvant conduire à terme à un effondrement de l'édifice trophique. Le cuivre présente donc selon la concentration et sa forme, des actions néfastes sur certains organismes en limitant leur croissance (par exemple : Hydrodictyon $s p$. et certaines cyanobactéries) mais représente aussi un oligo-élément essentiel à d'autres organismes (hémocyanine des crustacés et mollusques). Les espèces autochtones régressent inégalement en fonction de leur sensibilité, par contre, des espèces exotiques souvent invasives prolifèrent (moule zébrée, caridine, écrevisse américaine, nouvelle espèce de gammare tel que Dikerogammarus villosus, perche soleil) (Dembski et al., 2006, 2008; Maazouzi et al., 2007). Il est essentiel, en terme de santé publique, de remarquer que les poissons bioaccumulent le cuivre dans les viscères mais très peu dans le muscle qui est la partie consommée par l'homme (Vinot \& Pihan, 2005; Dembski et al., 2007). Les usages secondaires du site sont précisés par Dembski et al. (2005).

Selon Pronier (2000), les plans d'eau français, exposés à un climat tempéré présentent davantage d'écarts thermiques dus à l'altitude qu'à la latitude. Ils sont généralement 
monomictiques chauds en plaine et dimictiques en altitude. Les plans d'eau naturels sont caractérisés par un renouvellement relativement lent et continu de la masse d'eau, par un marnage réduit et par un faible indice de développement des berges associé à une forme subcirculaire de la cuvette. Dans les plans d'eau artificiels, la gestion hydraulique provoque un marnage plus ou moins ample, une discontinuité de l'écoulement vers le tributaire ainsi que des temps de séjour réduits (Poirel et al., 2001), enfin, le périmètre rapporté à la superficie du plan d'eau est élevé (Pronier, 2000). Le Mirgenbach représente donc un cas original. Ce plan d'eau artificiel de plaine présente un marnage limité, un périmètre conséquent, un court temps de séjour des eaux, une absence de stratification, une charge minérale élevée, un échauffement des eaux et une contamination métallique ayant une incidence directe sur des espèces appartenant au plancton et au benthos (Tab. I).

Le ruisseau du Mirgenbach présente un écoulement intermittent non compatible avec la vie piscicole dans le lieu d'implantation du réservoir. II ne peut être à l'origine de la présence de poissons dans le plan d'eau. Certaines populations de poissons ont pu coloniser le réservoir dès le remplissage par pompage en Moselle, en 1985. Les individus ont dû franchir les grilles et transiter par des conduites de plusieurs kilomètres depuis la Moselle jusqu'à la centrale puis de la centrale vers le réservoir (Tab. I). Des empoissonnements supplémentaires et contrôlés, issus d'étangs piscicoles lorrains concernent 4 espèces et 2 étapes distinctes (Dembski, 2005). En 1988 et 1989, les déversements ont eu lieu au stade de vésicule résorbée (brochet et sandre) et à l'âge d'un à trois ans (brochet, tanche Tinca tinca et carpe Cyprinus carpio). Depuis 2002 , le soutien de population a concerné le brochet au stade de fingerling. Le peuplement dans sa composition initiale (avant le raccordement des 4 tranches électronucléaires) dépend donc de la qualité physico-chimique des eaux de la Moselle au droit de la centrale, de l'introduction contrôlée d'espèces, des caractéristiques morphologiques du plan d'eau ainsi que de son usage industriel.

\section{MATÉRIEL ET MÉTHODES}

L'échantillonnage de poissons en domaine littoral est réalisé au moyen d'engins actifs, le microchalut et la senne de plage (Masson et al., 2001; Dembski, 2005). Le microchalut comprend un cadre métallique $(0,8 \mathrm{~m} \times 1,2 \mathrm{~m})$ supportant une poche en forme de chaussette dont la maille mesure $2 \mathrm{~mm}$. L'engin est tracté parallèlement à la rive sur une distance de $12 \mathrm{~m}$. La senne $(22 \mathrm{~m}$ de longueur $x$ $1,4 \mathrm{~m}$ de hauteur; maille de $2 \mathrm{~mm}$ ) comporte deux ailes symétriques disposées de part et d'autre d'une poche (3 $\mathrm{m}$ de profondeur) dans laquelle les poissons sont concentrés à la fin du trait. Les ailes, tout d'abord déployées parallèlement à la rive sont ensuite tractées en direction de la berge, de manière à encercler le poisson. Pour les deux types d'engins, un opérateur veille à dégager l'aire balayée de tout obstacle.

En domaine pélagique, les filets maillants verticaux sont déployés de 1991 à 2006, selon un protocole 
Tableau I. Caractéristiques du modèle Mirgenbach. Avantages (italique) et contraintes (souligné) pour l'étude des effets thermiques.

Table I. Mirgenbach's model characteristics : advantages (in italic) and constraints (underlined) for the study of the thermal effects.

\section{Caractéristiques Situation}

\section{Réservoir du Mirgenbach}

Remplissage

de la cuvette

Volume d'eau

Habitats littoraux
Poissons issus de la Moselle.

Empoissonnements volontaires identifiés.

Isolement des populations de poissons (grilles) pendant plusieurs générations.

Colonisation progressive par des espéces allochtones (zoobenthos en particulier).
Volume quasi constant, peu ou pas de marnage (fonctionnement normal).

Diversité d'habitats : modes exposés et abrités, pente douce à marquée, granulométrie et teneur en matiére organique variables.

Végétation terrestre rivulaire : strates herbacée, arbustive et arborée.

Végétation aquatique : hélophytes, herbiers.

Rives aménagées (digue, enrochements, gréve)

Apports et soutirages Renouvellement continu de la masse d'eau dans le Mrgenbach, apport et export d'eau continus de nutriments. Effet indéterminé pour les populations planctoniques.

\section{Brassage.}

Milieu non stratifié (fonctionnement normal), absence de thermocline et d'oxycline saisonniére.

Colonisation estivale de la colonne d'eau possible.

Absence de refuge thermique saisonnier.

$T^{\circ} \mathrm{C}, \mathrm{O}_{2}, \mathrm{pH}$, conductivité : peu d'hétérogénéité intrastationnelle ou interstationnelle instantanée.

\section{Effets du transit des eaux dans la centrale}

Rejets thermiques Milieu échauffé : température hivernale élevée, réchauffement " printanier" précoce, température estivale $<30^{\circ} \mathrm{C}$.

Évolution thermique saisonniére et interannuelle dépendante des conditions climatiques.

Évolution pluriannuelle lors du raccordement successif des tranches.

Rôle des Concentration des solutés par évaporation (incidence des aéroréfrigérants).

aéroréfrigérants Amplification de la charge minérale de la Moselle (dépendante du fond naturel et des activités anthropiques).

Évolution d'une situation mésotrophe vers un potentiel eutrophe.

Rôle des

Abrasion des conduites en laiton.

Conduites

Concentration cuprique croissante (eau, sédiment).

Effets écotoxiques sur la production primaire (phytoplancton) et les consommateurs primaires (zooplancton).

\section{Etudes et recherches}

Chronique disponible depuis 1986 (physicochimie des eaux, teneur en chlorophylle). Echantillonnage du poisson depuis 1987. Effort de pêche uniforme depuis 1991.

\section{Autres usages}

Pêche de loisir (Société de pêche depuis 1999), nautisme (planche à voile).

\section{Faculté d'étude des effets thermiques dans d'autres milieux}

Autres milieux lentiques (lacs)

Milieu lotique (eaux courantes)
Cuvette relativement stable en volume (inertie thermique), marnage modéré. Ecarts thermiques entre stations. Stratification thermique saisonniére.

Risque d'évolution saisonniére ou pluriannuelle du milieu physique. Hétérogénéité thermique transversale, longitudinale et verticale.

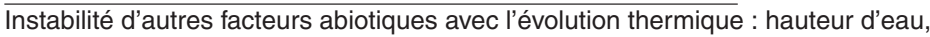
débit, teneur en oxygéne, connectivité latérale, continuité amont aval. 
standardisé. L'effort de pêche consiste dans la pose 24 heures consécutives, d'une batterie par station (1, 2, 3 et 4, Fig. 1), 2 fois par an : première campagne fin juin début juillet, seconde campagne en automne. Dans chaque station, la batterie comporte 6 filets dont les mailles mesurent 10, 20, 30, 40,50 et $60 \mathrm{~mm}$ de côté. La largeur des filets mesure $2,5 \mathrm{~m}$; leur hauteur correspond à la profondeur de la station (stations $1 \&$ \& $: 5 \mathrm{~m}$; stations $2 \& 3: 12$ et $15 \mathrm{~m}$ respectivement). Ces engins passifs comportent une nappe de filet en nylon monofilament; leur ralingue supérieure munie de flotteurs assure la flottaison tandis que la corde de fond légèrement lestée, repose sur le fond (Degiorgi \& Grandmottet, 1993; Degiorgi et al., 2001). Des échantillons récoltés entre février et mai de 1991 à 1993 puis de 2001 à 2006 permettent de compléter l'étude de la maturation sexuelle.

Les poissons marqués individuellement lors de la récolte aux filets maillants verticaux sont analysés frais ou après décongélation. Leur identification s'appuie sur l'observation de critères morphologiques externes et des dents pharyngiennes dans le cas des cyprinidés (Spillmann, 1961). Dans le cas des larves et alevins, les critères d'identification reposent en outre sur l'observation de la pigmentation (Urho, 1996; Pinder, 2001)

La longueur totale individuelle LT est mesurée au millimètre, du museau à l'extrémité de la queue; la précision de la pesée individuelle du poisson est de $0,1 \mathrm{~g}$. Après détermination du sexe, le développement sexuel est évalué par observation morphologique externe des gonades et par le calcul d'un indice, l'indice gonado-somatique (IGS) exprimant la part en pourcent de la masse des gonades dans la masse du poisson. La détermination de l'âge repose sur l'examen des opercules (sandre, perche) ou des écailles (perche soleil, cyprinidés) (Baglinière \& Le Louarn, 1987; Panfili et al., 2002 ; Bruslé \& Quignard, 2004).

\section{RÉSULTATS, DISCUSSION}

\subsection{Composition spécifique et contribution des espèces dominantes}

Dix huit espèces, au total, ont été recensées dans le plan d'eau, depuis sa création (Tab. II). Cette valeur s'approche de 20 qui est le nombre théorique d'espèces $(N)$ attendu dans le Mirgenbach, en raison de sa superficie $S$,

$$
\begin{aligned}
N= & 10,504 \times S^{0,1425} \\
& (\text { Barbour \& Brown, 1974), }
\end{aligned}
$$

cependant la diversité et la composition spécifiques n'ont cessé d'évoluer; ce peuplement du Mirgenbach peut être confronté à la typologie de Pronier (2000) établie dans 119 plans d'eau nationaux et à celle de Mehner et al. (2007) produite par l'analyse de 165 plans d'eau suédois, danois ou allemands, dont la structure des peuplements de poissons est établie au moyen de filets maillants scandinaves. Malgré, la différence des méthodologies adoptées, le Mirgenbach se situant à la limite de ces 2 territoires d'étude, les peuplements méritent être confrontés (Tab. II). En France, Pronier (2000) considère que l'altitude explique la structuration des peuplements ichthyologiques lacustres et que les gradients thermiques selon 


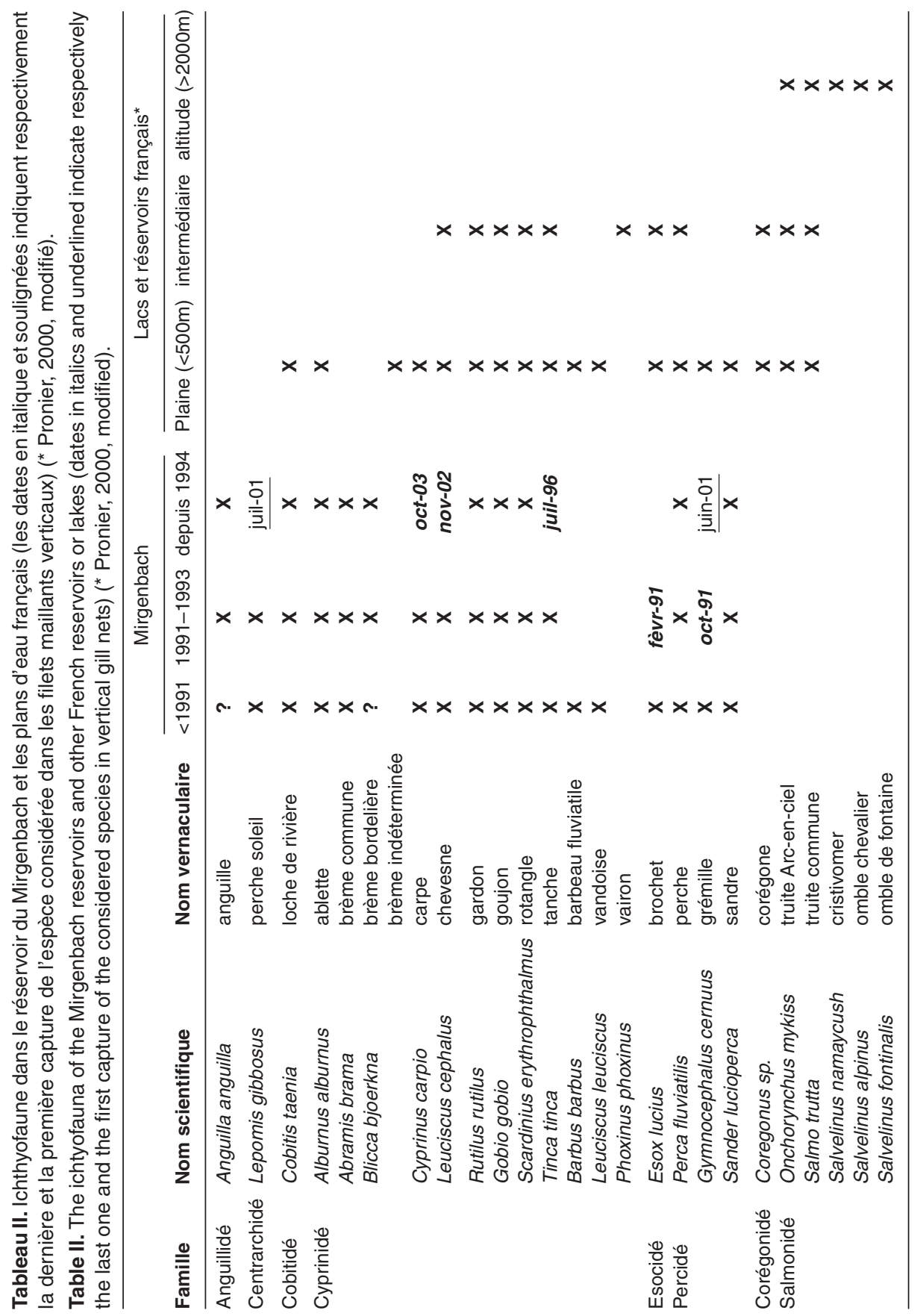


la longitude paraissent sans effet : 3 types de peuplement sont associés à trois plages d'altitude des plans d'eau délimités par les seuils de 500 et 2000 m (Tab. II). À l'inverse, Mehner et al. (2007) décèlent trois types d'assemblage variant selon l'altitude, mais aussi selon la latitude, la morphologie du plan d'eau ainsi que la richesse en certains nutriments. Les plans d'eau à corégones (Coregone albula) comportent des sandres Sander Iucioperca et des perches fluviatiles. Ils ont une faible teneur en phosphore total, une profondeur marquée, une petite dimension et caractérisent le nord-est de la région d'étude. Dans les plans d'eau à gardons, dont l'assemblage est également dominé par la perche fluviatile et le rotangle Scardinius erythrophthalmus, la dimension est réduite, la profondeur faible tandis que la concentration en phosphore total est élevée. Ces plans d'eau caractérisent le nord-est de l'écorégion étudiée. Enfin, dans le troisième type les plans d'eau à grémille Gymnocephalus cernua, accompagnée de la brème bordelière Blicca bjoerkna et de l'ablette Alburnus alburnus - les plans d'eau se caractérisent par un état intermédiaire et une répartition méridionale dans l'écorégion considérée. Dans le Mirgenbach, la dominance initiale successive des perches et des gardons, avec une charge en phosphore total élevée le rapproche du second type établi par Mehner et al. (2007). L'abondance actuelle des brèmes bordelières, la présence des grémilles et d'ablettes classerait le plan d'eau dans le type 3. Selon Pronier (2000), truites Salmo trutta et goujons Gobio gobio caractérisent des milieux frais et bien oxygénés. Le gardon, la perche et le brochet Esox lucius, espèces plus ubiquistes sont davantage sensibles à la qualité du milieu et à la température tandis que l'association d'espèces eurythermes que sont le sandre et la grémille caractérise les milieux plutôt turbides et peu exigeants en terme de qualité d'eau. Le Mirgenbach a évolué de l'un vers l'autre des deux derniers types d'association probablement sous l'effet conjugué de la température et de la trophie.

Le plan d'eau du Mirgenbach se caractérise par l'absence d'espèces sténothermes d'eau froide, absentes de la Moselle au droit de la centrale et caractéristiques des plans d'eau d'altitude mais également signalées dans les plans d'eau de plaine et les plans d'eau intermédiaires par Pronier (2000) (Tab. II). Parmi les espèces mésothermes, le brochet ne s'est pas maintenu. Parmi les espèces de Cyprinidés, les espèces rhéophiles normalement présentes dans les plans d'eau de plaine, le barbeau fluviatile Barbus barbus et la vandoise Leuciscus leuciscus ont disparu des captures depuis 1990. II faut noter la présence d'un poisson amphihalin thalassotoque, l'anguille (Anguilla anguilla) et l'absence de certaines espèces exotiques dont le silure Silurus glanis, présent dans la Moselle et particulièrement bien adapté à la vie en système lentique.

Les espèces dominantes récoltées aux filets maillants verticaux entre 1991 et 2006 sont par ordre de masse globale décroissante, la brème commune, le sandre, le gardon, la brème bordelière, la perche et la carpe. La stabilité de l'effort annuel de pêche $(2$ campagnes $\times 4$ stations $\times$ 6 engins $=1110 \mathrm{~m}^{2}$ de filet déployés/an) permet d'analyser l'évolution pondérale pluriannuelle. De 1991 à 2006, la masse annuelle cumulée 
des poissons tend à décroître (Fig. 2). De 101 et $168 \mathrm{~kg}$ (seuil maximal), récoltés respectivement en 1991 et 1992, la biomasse a chuté à 21 (seuil minimal) et $40 \mathrm{~kg}$, respectivement en 2005 et 2006.

Le déclin de la biomasse pourrait être provoqué par la raréfaction d'espèces, le changement de structure du peuplement, la diminution d'abondance (effectif des populations), la diminution de biomasse des populations soit par régression des cohortes les plus âgées soit par la diminution de croissance individuelle ou de croissance relative (relation entre longueur et masse).

Au cours de la chronique, chaque espèce est concernée par le déclin de biomasse. La perche initialement majoritaire (1986-1987; Flesch et al., 1994) occupe un rôle relativement accessoire depuis 1992 (Fig. 5). Le gardon (1990-1992) puis la brème

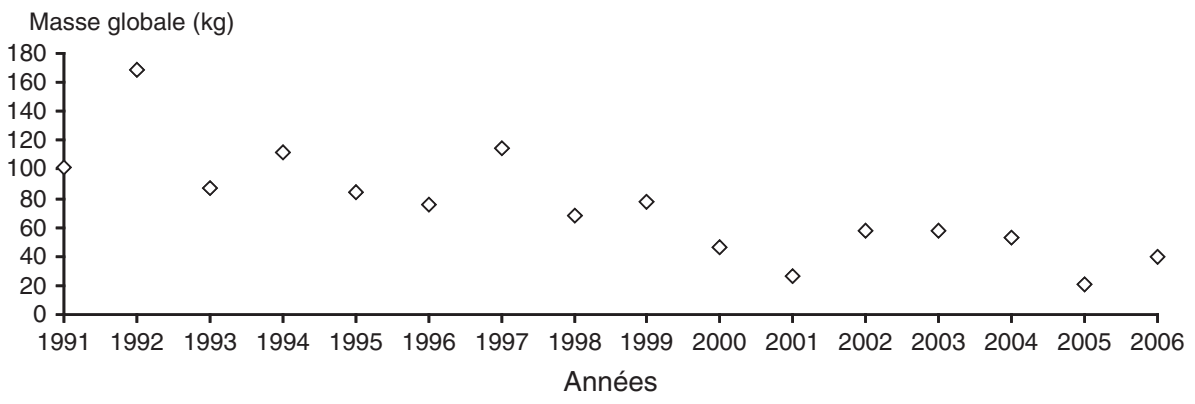

Composition pondérale relative (\%)

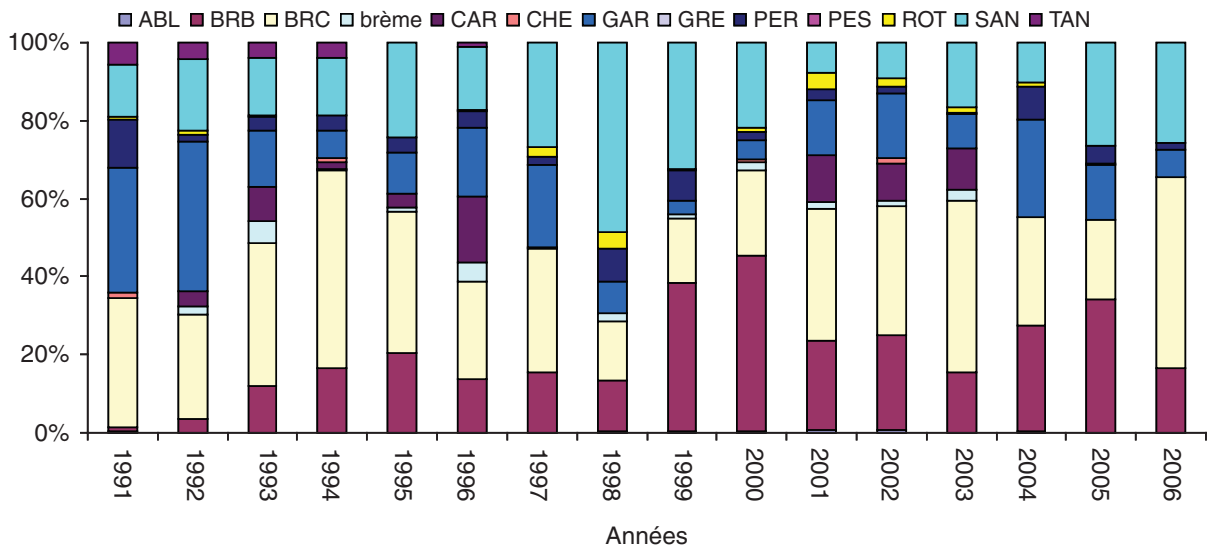

Fig. 5. Évolution des biomasses (en haut) et de la composition spécifique (en bas) des poissons récoltés aux filets maillants verticaux, dans le Mirgenbach (effort annuel de pêche : 2 campagnes $\times$ 4 stations $\times 6$ engins). Les abréviations sont précisées dans le tableau III.

Fig. 5. Variation of biomass (at the top) and specific composition (below) of harvested fishes by vertical gill nets in the Mirgenbach reservoir (annual effort of fishing: 2 seasons $\times 4$ stations $\times 6$ gears). See Table III for abbreviations. 
commune (depuis 1993) se sont alors succédés à la tête du peuplement. Depuis 1993, ce sont les mêmes espèces qui constituent l'essentiel de la biomasse récoltée : brème commune, sandre, brème bordelière, gardon si l'on exclut la capture ponctuelle de carpes de masse moyenne (>3 kg). La diminution de biomasse ne peut donc s'expliquer par le seul changement de composition faunistique du peuplement et la substitution d'espèces. Les autres indicateurs susceptibles de moduler la biomasse réclament une analyse de la structure démographique et des traits biologiques des populations.

$\mathrm{Au}$ sein des populations dominantes, la structure pondérale peut être évaluée en considérant lors des périodes initiale (1991-1993) et actuelle (2004-2006), deux classes pondérales réparties de part et d'autre du seuil de $500 \mathrm{~g}$ pour le sandre et de $100 \mathrm{~g}$ dans le cas du gardon, de la perche, de la brème commune et de la brème bordelière (Fig. 6). La faiblesse générale de l'abondance des petits individus en 1991 résulte de la précocité de la première campagne annuelle, limitant ainsi la capture de pré-recrus (jeunes de l'année entrant dans le domaine pélagique). La distribution pondérale varie selon les espèces (Fig. 6). Dans le cas de la perche, les captures déclinent pendant la période initiale (1991-1993); les masses récoltées restent faibles de 2004 à 2006 et la population vieillit si l'on considère le déclin d'abondance des moins gros poissons. Dans le cas du gardon, ce phénomène est différé, car la biomasse est maximale en 1992. La biomasse des brèmes communes décroît entre la période initiale (1991-1993) et récente (2004-2006) avec une augmentation notable de la classe des poissons de masse inférieure à $100 \mathrm{~g}$. La tendance est comparable dans le cas de la brème bordelière dont la part des poissons de la classe inférieure tend à progresser en masse et en effectif. Le sandre est marqué par un déclin de la masse globale entre 19911993 et 2004-2006, ainsi que par une réduction progressive des représentants de la classe inférieure $(<500 \mathrm{~g})$.

\subsection{Traits biologiques des poissons du Mirgenbach}

\subsubsection{Effets directs de la température} sur le calendrier de développement

La période de reproduction des espèces printanières (sandre, perche, gardon et brème commune) est précoce dans le Mirgenbach où l'on atteint très tôt la température favorable à la ponte de chaque espèce (Tab. III). Dans la rivière Meuse, un accroissement de 2 à $3^{\circ} \mathrm{C}$ dû à des rejets thermiques accélère la gamétogenèse et induit une maturation sexuelle plus précoce du gardon (Mattheeuws et al., 1981). Dans le Léman, l'accroissement de $2{ }^{\circ} \mathrm{C}$ constaté en mai, entre 1986 et 2000 expliquerait l'avancement de sa reproduction d'une quinzaine de jours (Gillet \& Quétin, 2006). La maturation finale du gardon est atteinte avant le seuil de $1500^{\circ} \mathrm{C}$ jours, le calcul débute le $1^{\mathrm{er}}$ octobre et tient compte d'un seuil minimal de $3^{\circ} \mathrm{C}$ nécessaire à sa maturation (Gillet \& Quétin, 2006). Depuis I'hiver 1991/1992, postérieurement au raccordement des 4 tranches, ce seuil de $1500^{\circ} \mathrm{C}$ jours est atteint dans le Mirgenbach, avant la fin du mois de février, voire 

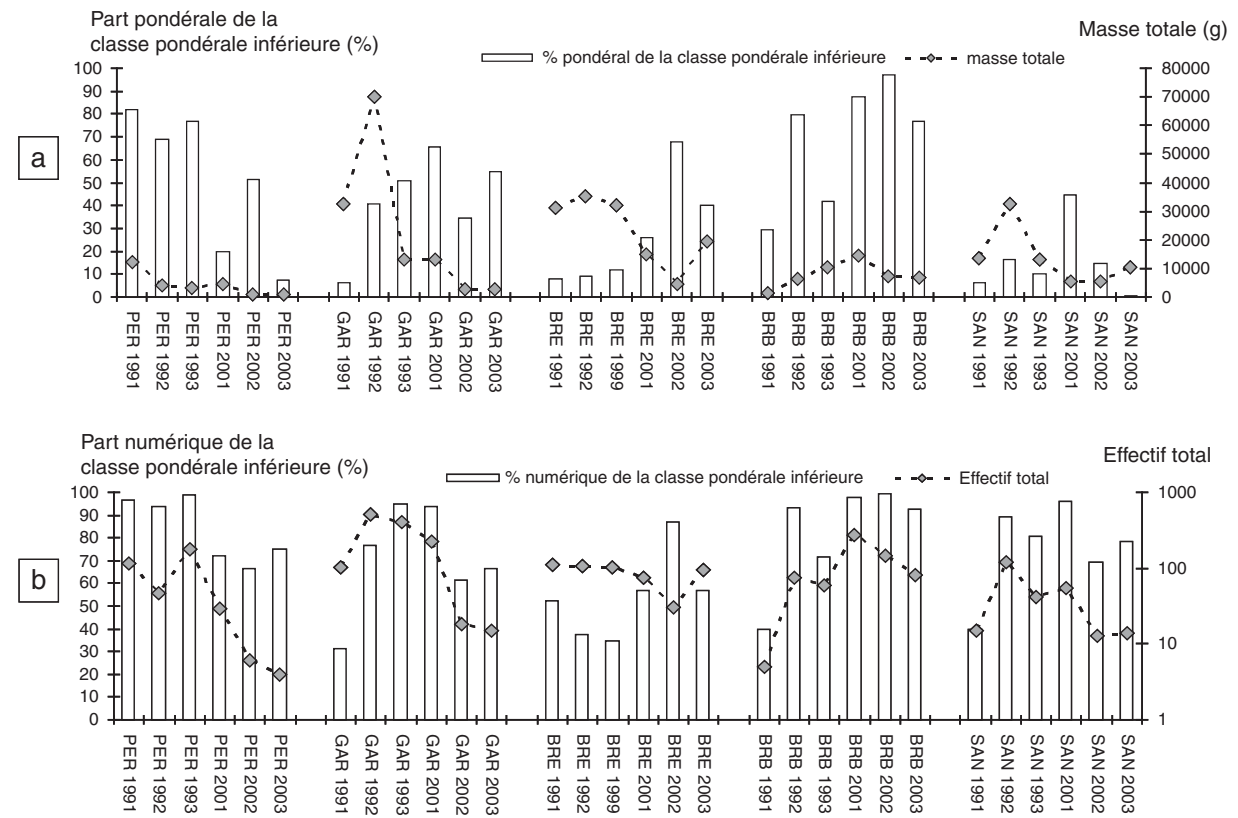

Fig. 6. Évolution temporelle de la structure pondérale des captures de perche (PER), de gardon (GAR), de brème commune (BRE), de brème bordelière (BRB) et de sandre (SAN) (pêche aux filets maillants verticaux). Classe pondérale inférieure : perches, gardons, brèmes communes et bordelières de masse individuelle inférieure à $100 \mathrm{~g}$; sandres de moins de $500 \mathrm{~g}$ (La classe supérieure représente le complément). (a) Évolution des masses totales (axe droit) et de la part pondérale relative de la classe pondérale inférieure dans les captures (axe gauche). (b) Évolution des effectifs totaux (axe droit) et de la part numérique relative de la classe pondérale inférieure dans les captures (axe gauche).

Fig. 6. Temporal weight structure variation of the captures of perch (PER), roach (GAR), bream (BRE), silver bream (BRB) and pike-perch (SAN). Inferior weight class (Classe pondérale inférieure: $<100 \mathrm{~g}$ for perch, roach, bream and silver bream, $<500 \mathrm{~g}$ for pike-perch). (a) Variation of total mass (right axis) and relative part of the inferior weight class (left axis: \% biomass, histogram). (b) Variation of the total number (right axis) and the relative part of the inferior weight class (left axis: \% number, histogram).

avant la mi-février. En 1987, la première tranche étant raccordée en automne 1986, ce seuil était atteint le 10 mai; cette date a évolué entre 1987 et 1991 avec le raccordement des tranches successives jusqu'en automne 1991. Dans le Mirgenbach, la croissance et le développement individuels sont favorisés par cette reproduction précoce; les jeunes de l'année vont bénéficier à date équivalente, d'un plus grand nombre de degrés jours que dans les autres milieux aquatiques régionaux. Ce phénomène est également signalé dans le réservoir Ivan'Kovo (centrale de Konakovo; Boystov, 1971). Dans le Mirgenbach, les alevins accomplissent précocement leur développement dans les habitats littoraux pour rejoindre 


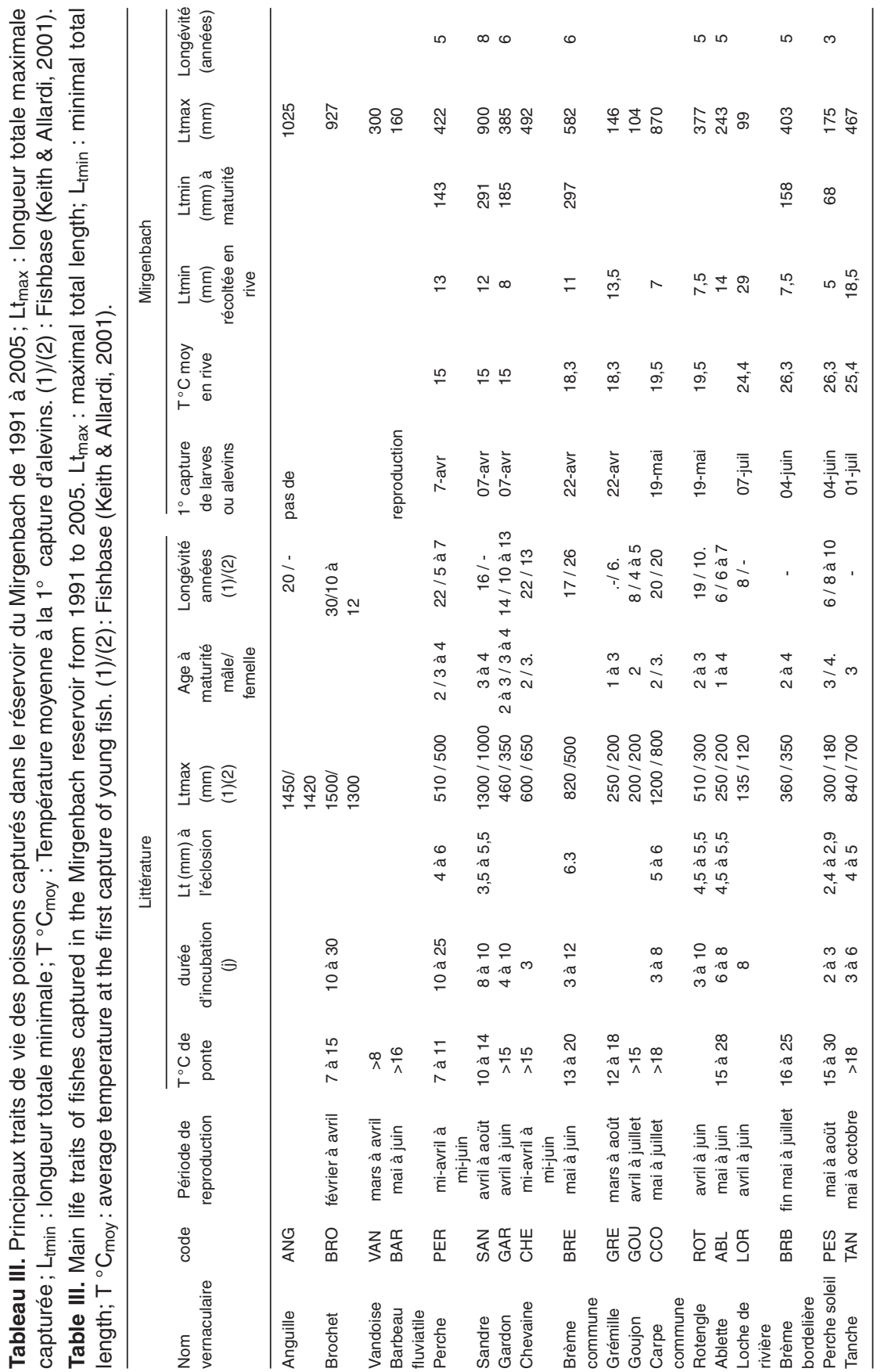


ensuite le domaine pélagique. En juillet, lors des campagnes aux filets maillants verticaux, les gardons, les perches, et les sandres mesurent respectivement de l'ordre de 8, 11, et $10 \mathrm{~cm}$ (année 1999, Dembski, 2005). En l'absence de stratification thermique saisonnière l'ensemble de l'espace leur est potentiellement accessible. Cependant, les aptitudes spécifiques varient. En début d'été, les jeunes perches et gardons vivent respectivement entre 1 et $5 \mathrm{~m}$ de profondeur et de la surface à $7 \mathrm{~m}$ de profondeur tandis que les sandres colonisent l'ensemble de la colonne d'eau, y compris les parties les plus profondes (Dembski, 2005). En automne, les poissons continuent à bénéficier de conditions de croissance favorables avec l'abaissement tardif de la température.

\subsection{2 Évolution pluriannuelle des traits biologiques des jeunes de l'année}

Les caractéristiques évoquées ne sont cependant pas stables au cours de la chronique. Dans le Mirgenbach, entre 1990 et 1991, la perche se reproduit en mi-mars, à une température de $12^{\circ} \mathrm{C}$ alors que la fraye est signalée mi-avril dans les étangs piscicoles régionaux (Flesch, 1994). En 2003, cette température seuil de $12{ }^{\circ} \mathrm{C}$ est atteinte dès fin février avançant ainsi le calendrier de reproduction. De même, en domaine littoral, la taille moyenne des gardons de l'année augmente entre le $07 / 05 / 1992(\mathrm{LT}=8$ à $10 \mathrm{~mm}$ ) et le 02/05/2002 (LT = 10 à $21 \mathrm{~mm}$, LT médiane $=15 \mathrm{~mm}$; test $\mathrm{t}, \mathrm{p}<0,01)$. Cet écart de taille se maintient en domaine pélagique (pêche aux filets maillants verticaux). La majorité des gardons mesurent de 72 à $77 \mathrm{~mm}$, le 2 juillet 1993 et entre 80 et $84 \mathrm{~mm}$, le $1^{\text {er }}$ juillet 1997 (Test t, $\mathrm{p}<0,01$ ) (Dembski et al., 2007).

Pour la brème commune, si les jeunes de l'année sont régulièrement capturés au moyen des filets maillants verticaux en domaine pélagique, lors de la campagne automnale depuis 1991, ceux-ci apparaissent pour la première fois en 1997 et systématiquement depuis 2000, dans les captures estivales (Dembski, 2005). Or ces jeunes brèmes communes sont capturées l'été avec la maille de $10 \mathrm{~mm}$ dont l'unité d'effort est constante depuis 1991. Cette modification du régime des captures peut alors s'expliquer par une avancée de la date de reproduction de cette espèce dont les alevins migreraient en domaine pélagique plus tôt dans la saison que les années précédentes et seraient alors vulnérables aux filets maillants dès le début de l'été. Toutefois, on n'observe aucune modification notable du régime thermique au cours de cette période (comme par exemple un réchauffement printanier plus précoce) susceptible d'expliquer ce décalage de la reproduction. On suppose alors que les jeunes brèmes communes ont soit bénéficié d'une croissance plus forte leur permettant de quitter les rives plus tôt dans la saison, soit quitté les rives en raison d'une exclusion causée par l'arrivée d'une espèce compétitrice (la brème bordelière est en plein essor et la perche soleil prolifère depuis 2000), soit enfin bénéficié d'un espace vacant au large en raison d'un déclin du prérecrutement des perches, gardons et sandres de l'année (Dembski, 2005).

$\mathrm{Au}$ cours de la chronique pluriannuelle, postérieurement à la mise en 
fonction de la dernière tranche de production énergétique, il semble donc que l'on assiste à la fois à un décalage temporel des calendriers de reproduction puis de développement des jeunes de l'année, et à un changement de la structure estivale précoce (fin juin-mi juillet) du peuplement de jeunes de l'année en domaine pélagique où les brèmes communes se sont substituées aux cohortes en déclin, de jeunes perches, gardons et sandres. Cette précocité de l'abandon du domaine littoral par ces alevins favorise le développement démographique de la perche soleil dont les jeunes de l'année prolifèrent dans un espace relativement délaissé par les autres espèces à leur naissance (période de reproduction : mois de juin à août); à partir de septembre, elles représentent plus de $90 \%$ des effectifs de jeunes de l'année (Dembski et al., 2008). Au stade adulte et en phase de maturation sexuelle, les perches soleil partagent l'espace littoral avec les géniteurs des différentes espèces qui se succèdent pendant la fraye, de la fin de l'hiver au début de l'été (Dembski, 2005).

II sera nécessaire de confronter les indicateurs de croissance et d'abondance des jeunes de l'année avec les indicateurs mésologiques pour disjoindre les effets d'échelles temporelles variables (évolution thermique dépendante de la saisonnalité, du changement global et des conditions d'exploitation du site). Ainsi, dans le Lac Windermere (Grande Bretagne), la croissance des perches de moins d'un an est corrélée aux mouvements du Gulf Stream au sein de l'océan Atlantique; les tailles moyennes les plus faibles entre 1966 et 1990 étant associées aux déplacements les plus septentrionaux. II est probable que la position du Gulf Stream agisse sur les conditions climatiques locales, notamment le vent dont on connaît les effets sur la stratification et le développement planctonique printanier et estival (Winfield et al., 1998).

\subsection{3 Évolution pluriannuelle des traits biologiques des adultes}

Les poissons du Mirgenbach ont pu atteindre des longueurs maximales remarquables (Tab. II) (Marzou, 1996; Dembski, 2005). Cependant le fait marquant concerne la diminution progressive de ce descripteur au cours de la chronique. Quelques exemples de réduction de ces tailles maximales peuvent être rapportés en confrontant les valeurs rencontrées en début et en fin de chronique (Iongueur totale en mm entre 1991 et 1993 / puis de 2004 à 2006) : brème commune (582/393), brème bordelière $(403 / 315)$, ablette $(243 / 160)$, gardon $(385 / 333)$ rotangle $(377 / 310)$, perche $(400 / 345)$. Si la diminution pluriannuelle des longueurs maximales n'affecte pas l'ensemble des espèces (grémille : 146/167; sandre : 811/918), par contre l'abaissement de la longévité les concerne toutes. Ainsi pour les espèces les mieux représentées, entre 1991 et 2002, l'âge maximal a chuté de 8 à 3 ans pour le gardon, de 6 à 4 ans pour la brème commune, de 5 à 3 ans pour les brèmes bordelières.

L'âge de recrutement qui marque l'entrée des jeunes dans le stock des adultes est relativement précoce dans le Mirgenbach. En 1991, les perches mâles sont adultes à $13 \mathrm{~cm}$ dés l'âge d'un an; les plus petites femelles adultes mesurent $14 \mathrm{~cm}$, à l'âge de 2 ans (Flesch, 1994). Dans le cas de 
la perche soleil, la maturité sexuelle est acquise à l'âge d'un an pour les mâles et les femelles. II n'est pas capturé de poisson immature âgé d'un an ou plus pendant la période de reproduction (Dembski et al., 2006; Valente, 2008). La maturité sexuelle est plus tardive dans l'aire de répartition européenne et dans le continent d'origine (Tab. II). Dans le cas du gardon, des brèmes commune et bordelière, l'âge et la taille de maturité sexuelle, déjà précoces au début des années 1990 diminuent durant la chronique (Tab. III). Parallèlement, la réduction de taille des plus petits poissons matures et des tailles maximales capturées peut être interprétée comme étant la manifestation d'une diminution de taille des plus petits et des plus grands géniteurs. Ces résultats expliquent l'évolution de la structure pondérale de certaines populations, évoquées précédemment (Fig. 6). La diminution progressive de la biomasse globale de poisson durant la chronique (Fig. 5) s'explique donc par les variations d'abondance, c'est-à-dire le déclin de populations en raison de la réduction du renouvellement des populations (perche, sandre, gardon) et pro parte par le changement de structure pondérale des populations et de la diminution numérique et pondérale des plus grands gardons, brèmes communes et bordelières. Cet abaissement de longévité limite le nombre de cohortes participant à la reproduction ; la réduction des longueurs maximales ou du volume de capture des plus gros poissons abaisse en outre, la masse des géniteurs les plus féconds. Cette évolution fragilise le renouvellement des populations, notamment en cas de conditions climatiques défavorables pendant la phase de reproduction.

\subsection{Gradients thermiques et autres causes de variations des traits}

Au sein de l'aire biogéographique d'une espèce, les traits biologiques sont susceptibles de varier selon des gradients thermiques de latitude, de longitude ou d'altitude (Bruslé \& Quignard, 2004). La littérature rapporte notamment des écarts de longévité et d'âge de maturité (l'épinoche par exemple; Bruslé et Quignard, 2004), ainsi que des Iongueurs minimales et maximales des géniteurs (cas de la perche soleil; Villeneuve et al., 2005). Cette plasticité s'exprime généralement sous l'influence de la température moyenne annuelle dont la valeur dans les régions septentrionales ou orientales ralentit l'activité métabolique et le développement individuels. Cependant, la phénologie ne se décline pas nécessairement dans les mêmes conditions de photopériode (décalage des calendriers de reproduction), ni de température. Chez la perche, les populations septentrionales se reproduisent à des températures inférieures à celles des populations méridionales (Thorpe, 1977). À l'échelle de son aire de répartition biogéographique européenne (aire d'introduction), le potentiel invasif de la perche soleil peut être prédit par le simple constat d'une relation entre la température, la croissance des juvéniles et l'âge de maturité (Copp et al., 2004). Dans l'aire méridionale et l'est de l'Europe, sous l'effet de la température, les perches soleil manifestent un caractère proliférant provoqué par une croissance rapide et une maturation sexuelle précoce. À l'inverse, les populations septentrionales vivant dans des eaux plus froides connaissent une croissance 
plus lente, une maturation sexuelle plus tardive et ne connaissent pas de phénomène proliférant. L'échauffement dans le Mirgenbach induit une amplification de l'effort de reproduction (adultes jeunes, mâles et femelles aux IGS élevés) qui se traduit par une prolifération des jeunes perches soleil de l'année (Dembski et al., 2006, 2008; Valente, 2008). Ce potentiel ou cette stratégie représente certains risques pour les espèces natives. A contrario, le défaut d'espèces lacustres adaptées à ces conditions thermiques offre un espace littoral vacant à la perche soleil (Irz et al., 2004a, 2004b; Dembski, 2005 ; Dembski et al., 2006, 2008).

Au sein d'une même population, sous l'effet d'une exploitation halieutique, l'âge et la taille de maturité déclinent; ce constat est observé en milieu marin, en quelques décennies pour la morue Gadus morhua, dans le Nord-est et le Nord-ouest Atlantique ainsi qu'en mer Baltique (Law, 2001). Ce phénomène concerne également la plie Pleuronectes platessa dans la mer du Nord, depuis le début du vingtième siècle (Law, 2001). Cette évolution répond à la réduction de biomasse par capture des plus grands organismes dont la disparition favoriserait l'accès à la nourriture pour les plus jeunes classes d'âge; les individus bénéficieraient alors d'une croissance individuelle plus forte et atteindraient la taille adulte à un âge plus précoce. La sélection génétique pourrait également être la cause de cette précocité de l'âge adulte. L'environnement et son évolution naturelle ou l'action indirecte de l'homme peuvent également contribuer partiellement à ce changement. Que l'on considère une espèce particulière ou l'ichthyocénose, le renouvellement des générations et l'effort de reproduction qui sont des éléments clés du taux d'accroissement des populations, sont euxmêmes corrélés à plusieurs traits biologiques facilement accessibles que sont la taille et l'âge à maturité, le taux de croissance individuelle et les tailles en présence (Reynolds et al., 2001). Ainsi, à l'intérieur de la plupart des familles de poissons, le rapport entre la longueur de première maturité sexuelle et la longueur asymptotique est relativement constant (Pauly \& Moreau, 1997). Dans le Mirgenbach, l'abaissement conjoint des longueurs maximales rencontrées et de l'âge de maturité évoque ce phénomène. Les dynamiciens utilisent notamment le modèle de von Bertalanffy, pour obtenir des traits biologiques dépendants des longueurs maximales et de la longévité (Reynolds et al., 2001). Cette approche devra être développée dans le Mirgenbach pour lequel la modélisation de la croissance concerne un nombre réduit d'espèces et des données non actualisées (perche : Flesch, 1994; carpe d'élevage : Dubost, 1996 ; gardon : Marzou, 1996).

Lorsque les conditions trophiques ne sont pas limitantes, la croissance ou les fonctions biologiques qui la conditionnent, dont la synthèse protéique, dépendent de la température (McCarthy \& Houlihan, 1997 ; Jobling, 1997). La croissance est donc relativement prédictible. Ainsi, l'étude de la croissance de la morue menée pendant plusieurs décennies, révèle une relation directe entre la température et la masse corporelle à l'âge de 4 ans (Ouest du Groenland et îles Feroé; Brander, 1996). Pour cette espèce, il 
Tableau IV. Évolution de traits biologiques du gardon, de la brème bordelière et de la brème commune dans le réservoir du Mirgenbach.

Table IV. Biological traits evolution for roach, silver bream and bream from the Mirgenbach reservoir.

\begin{tabular}{|c|c|c|c|c|c|c|c|}
\hline \multirow[b]{2}{*}{ espèce } & \multirow[b]{2}{*}{ code } & \multicolumn{2}{|c|}{$\begin{array}{l}\text { Age de recrutement } \\
\text { (années) }\end{array}$} & \multicolumn{2}{|c|}{$\begin{array}{l}\text { Longueur totale } \\
\text { minimale à la } 1^{\circ} \\
\text { maturité }(\mathrm{cm})\end{array}$} & \multicolumn{2}{|c|}{$\begin{array}{l}\text { Longueur maximale } \\
\text { rencontrée }(\mathrm{cm})\end{array}$} \\
\hline & & avant 1993 & depuis 2004 & avant 1993 & depuis 2004 & avant 1993 & depuis 2004 \\
\hline Gardon & GAR & 2 & 2 & 20 & 16 & 38 & 34 \\
\hline $\begin{array}{l}\text { Brème } \\
\text { bordelière }\end{array}$ & BRB & 3 & 2 & 23 & 17 & 38 & 26 \\
\hline $\begin{array}{l}\text { Brème } \\
\text { commune }\end{array}$ & BRE & 3 & 2 & 35 & 29 & 58 & 37 \\
\hline
\end{tabular}

est possible de construire un modèle logistique reliant la masse individuelle à un âge donné (entre 2 et 9 ans) et la température à laquelle l'animal a été exposé (température $\times$ âge) avant sa capture. Dans le Mirgenbach, la croissance des immatures est facilitée à la fois par le décalage du calendrier de reproduction et par le bilan thermique annuel. La maturation sexuelle précoce paraît en être une conséquence ; la contrepartie pourrait en être la faible longévité. Dans le cas des brèmes commune et bordelière ainsi que du gardon capturés au cours des dernières années, la lecture des écailles révèle un gain de croissance linéaire encore plus important pendant la seconde année que lors de la première année. Au-delà du deuxième hiver, alors que les individus connaissent un développement sexuel, les stries hivernales sont beaucoup plus rapprochées et ne progressent visiblement pas selon le même taux d'accroissement, marquant ainsi une forte discontinuité entre les stades juvénile et adulte. L'observation des écailles de perche soleil ne conduit pas au même constat, les animaux semblent croître pendant leur développement sexuel printanier (étude en cours). Il sera nécessaire de confronter les conditions de croissance pour les poissons immatures et adultes des cohortes successives afin de vérifier la stabilité des conditions de croissance des immatures et le déclin progressif de la croissance et des longueurs maximales des gardons et brèmes commune ou bordelières adultes.

\subsection{Cas des populations exposées à un échauffement durable}

Qu'en est-il de l'évolution des traits biologiques dans le cas de populations soumises à un échauffement? En Suède, dans la mer Baltique, l'exposition des perches aux eaux de rejet de la centrale électronucléaire de Forsmark provoque une maturation sexuelle à un âge plus précoce et l'accroissement de la mortalité des adultes, ce qui déclenche le déclin de la part des poissons les plus âgés (Van der Kraak \& Pankhurst, 1997). En Lituanie, dans le lac Druksiai modifié en réservoir, le gardon connaît un gain de croissance linéaire et pondéral graduel lors du couplage du plan d'eau à 
la centrale électronucléaire d'Ignalina (Balkuviené \& Pernaraviciuté, 1994). Parallèlement au changement de température, la longévité de cette espèce décline, évoluant de 18 à 14 ans. Dans un réservoir connaissant un impact similaire (effluents thermiques) en Lituanie (Rivière Streva, Lac Anikstas), Gajdusek et al. (1987) montrent que les brèmes communes ont connu une diminution des longueurs maxima et des masses individuelles (divisées par deux pour les poissons les plus âgés), une baisse de longévité passant de 15-18 ans en conditions initiales, à 10 ans par la suite. Par ailleurs l'âge de recrutement a diminué tant que des conditions trophiques favorables se sont maintenues.

L'évolution temporelle de certains traits biologiques semble donc être une constante dans les milieux échauffés, tout comme dans le cas des populations marines exploitées précédemment évoquées (morue, plie). Dans le Mirgenbach, depuis l'automne 1991, les populations de poissons sont exposées à un régime thermique saisonnier relativement stable, alors que les conditions trophiques ne cessent d'évoluer, avec la réduction de la biomasse chlorophyllienne. La production individuelle du poisson correspond au bilan de prise et de dépense d'énergie. Ce bilan évolue avec la température et la ressource alimentaire (Jobling, 1994, 1997). II décroît et peut devenir négatif lorsque les dépenses cataboliques ne sont pas compensées par une ration alimentaire adéquate. La disparition précoce des animaux (Iongévité réduite) pourrait-elle en être la conséquence? II faut en effet se demander si la température et le taux cuprique élevé ne combinent pas leurs effets en une variable de forçage métabolique; le cuivre limitant peut être la production primaire et ayant un effet écotoxique sur le zooplancton, le niveau d'activité métabolique induit par la température ne serait pas toujours compensé par une ressource alimentaire accessible, ni compensé par la disponibilité en tissus de réserve.

Plusieurs remarques s'imposent alors. La diminution parallèle de la biomasse chlorophyllienne et de la biomasse de poisson traduirait une dépendance de plus en plus pesante des poissons à l'égard d'une ressource trophique en déclin. Toutes les espèces et tous les stades seraientils uniformément affectés par le défaut de ressource? Le déclin des effectifs de jeunes constaté dans les pêches annuelles aux filets maillants verticaux, est-il la conséquence d'un défaut de développement des jeunes par densité dépendance, ou bien ce déclin d'abondance est-il le résultat d'une diminution d'efficacité de la reproduction, de l'abondance ou de la biomasse des géniteurs? Les conditions saisonnières de développement du zooplancton pourraient avoir changé en raison de contraintes trophiques et cupriques et devront être examinés. L'existence de relations entre biomasse des adultes et volume des pré-recrus (au sens de l'entrée dans le domaine pélagique) mériterait d'être évaluée en utilisant le jeu de données des captures aux filets maillants verticaux. La rupture de croissance ou d'abondance constatée à partir du recrutement ainsi que la faible longévité des adultes pourraient être imputées à l'effort de reproduction. Les adultes affaiblis se rétabliraient difficilement après la fraye ou ne survivraient pas au développement 
sexuel. Un examen approfondi des relations entre longueur et masse individuelles et de l'indice de condition devrait permettre d'évaluer l'embonpoint des animaux selon leur stade et la stabilité ou non de l'indice au cours de la chronique. Pronier (2000) observe une convergence de résultats entre la qualité de l'indice de condition (valeurs élevées) et les indicateurs d'abondance de gardons dans un échantillon de milieux lacustres français.

La croissance est limitée par la teneur en oxygène du milieu et surtout par la surface des branchies qui grandissent moins vite que le volume du poisson qu'elles approvisionnent en oxygène (Pauly \& Moreau, 1997). Au fur et à mesure de la croissance, la consommation relative d'oxygène diminue jusqu'à une telle masse infinie que la croissance individuelle s'arrête parce que la quantité d'oxygène absorbé suffit juste à assurer le métabolisme de maintien. Ainsi dans une population donnée, les mécanismes telle que l'élévation de la température qui contribuent à accroître le métabolisme de base et la consommation d'oxygène entraîneront une diminution de la taille et de la masse maximales.

Le Mirgenbach est un milieu bien oxygéné. Le déclin de la biomasse chlorophyllienne après 1992 provoqué par la contamination cuprique ou par la diminution de l'ensemencement algal provenant des eaux de la Moselle suggère une diminution de la ressource trophique accessible aux consommateurs primaires. En conséquence, ce déclin contrôlerait et limiterait la biomasse des prédateurs poissons. En Lac, la production de poissons est corrélée avec la production primaire (Downing et al., 1990). En outre, dans le Mirgenbach, pour rechercher et récolter leurs proies devenues plus rares, les poissons doivent être plus actifs et accroissent leur dépense métabolique. D'après Pauly et Moreau (1997), cette consommation supplémentaire d'énergie et d'oxygène réduirait les capacités de l'espèce à se maintenir à leurs longueurs et masses maximales initiales. Dans le Mirgenbach, cette hypothèse expliquerait donc la convergence entre les réductions de la biomasse chlorophyllienne, de la masse individuelle maximale chez de nombreuses espèces et de la biomasse globale des poissons. Ces éléments confortent la théorie d'une relation forte entre traits biologiques telle que le taux d'accroissement individuel (ici sous le contrôle de la température) et le taux d'accroissement des populations (Reynolds et al., 2001).

D'après la littérature, les espèces ne manifestent pas les mêmes réactions, ni les mêmes risques lorsque les adultes sont exposés à l'échauffement des eaux. Une étude de l'ovogénèse menée dans plusieurs sites soumis aux rejets caloriques, conduit les auteurs à considérer que pour les espèces à reproduction estivale (brème bordelière, grémille), les effets thermiques semblent peu prononcés (Luksiene et al., 2000); par contre, les espèces à reproduction printanière (brochet, perche et gardon) sont faiblement adaptées à des conditions d'échauffement en automne, en hiver et au printemps. Ainsi, chez la perche, la perturbation du développement ovarien affecte une forte majorité des plus grands individus, les empêchant probablement de participer à la reproduction du cycle immédiat. Un constat équivalent est dressé pour le brochet. Chez le gardon, la 
maturation ovocytaire est synchrone. Le développement ovarien commence au début de l'automne. La vitellogenèse progresse lentement pendant l'hiver, au moins tant que la température paraît dépasser $3^{\circ} \mathrm{C}$; la ponte est printanière (Rinchard \& Kestemont, 1996; Gillet \& Quétin, 2006). Sur les sites d'Ignalina, d'Oskarshamn et de Forsmark, les gardons subissant l'exposition aux rejets thermiques connaissent des risques de développement asynchrone, de ponte partielle et de dégénérescence ovocytaire (Luksiene et al., 2000). Ce phénomène connu à l'état naturel est amplifié dans les systèmes échauffés. Des cas d'hermaphrodisme sont rapportés. Dans le Mirgenbach, l'étude histologique des perches soleil révèle un cas unique de cette nature pour plusieurs centaines d'observations (Valente, 2008). L'ensemble des phénomènes rapportés est attribué à des dysfonctionnements hormonaux et à des difficultés d'ordre métabolique (Luksiene et al., 2000). La perche par exemple pourrait s'épuiser par défaut de consommation alimentaire soit parce que la température est excessive au début de la phase de maturation sexuelle, soit parce qu'une longue scotophase automnale et hivernale limiterait l'efficacité de la chasse à vue. Dans le Mirgenbach, ces contraintes combinées d'allocation énergétique (prise alimentaire, effort de reproduction) et de défaut ou de déclin d'efficacité de la reproduction expliqueraient donc le déclin précoce et progressif des populations de perche et de gardon et enfin la régression différée des populations à reproduction plus tardive (brèmes commune et bordelière, sandre, perche soleil) et/ou moins affectées par l'échauffement (grémille).
Les populations continuent à subir l'effet de facteurs contrôlant qu'ils soient stabilisés (la température) ou non (contamination cuprique, variation de la qualité d'eau de la Moselle amont), dans un contexte de renouvellement continu de la masse d'eau, ce qui confère une profonde immaturité et instabilité au système et complique son analyse. Les brèmes bordelières désormais très jeunes, les brèmes communes dont les plus grandes femelles ne paraissent pas toutes en état de se reproduire (IGS faibles) dénotent que les populations thermophiles subissent des pressions de sélection sévères. Les poissons qui débutent leur maturation sexuelle annuelle en fin d'été et début d'automne (perche, sandre, gardon, brèmes commune et bordelière, notamment) paraissent donc plus en difficulté que la perche soleil dont la maturation commence au cours de l'hiver (Dembski, 2005; Dembski et al., 2006; Valente, 2008).

Le Mirgenbach constituerait donc un modèle particulier pour l'étude des effets d'un changement thermique puisqu'il soumet des populations isolées à un double effet, celui d'un échauffement hivernal marqué qui limiterait en particulier les capacités de reproduction des espèces non thermophiles et, celui d'une ressource trophique limitante agissant sur la masse individuelle et la biomasse globale.

\section{CONCLUSIONS ET PERSPECTIVES}

Le gradient de productivité des eaux continentales (Persson et al., 1991), mesuré à l'aide de la biomasse de chlorophylle conduit à une 
succession du type Salmonidés remplacés essentiellement par la perche auxquels se substituent les cyprinidés. Dans le Mirgenbach, la perche a été remplacée par le gardon lui-même supplanté par les brèmes communes et bordelière. Malgré la réduction de la biomasse chlorophyllienne, cette évolution s'est accentuée; la température pèserait alors davantage que les conditions trophiques dans la structuration de l'ichthyocénose.

La température agit sur le métabolisme individuel et module la phénologie : le calendrier de développement et les étapes de colonisation des domaines littoraux et pélagiques au cours du cycle annuel et du cycle vital paraissent interdépendants. Lorsque les animaux ont atteint l'âge adulte leur survie paraît altérée; le renouvellement des générations est mis en péril par la diminution progressive de la masse individuelle et globale de géniteurs qui accompagne le déclin de longévité. L'évolution des traits individuels est continue pendant la chronique, ce qui modifie profondément la structure des populations. $\mathrm{Au}$ sein du peuplement, les espèces mésothermes telles que le brochet, le gardon et la perche ont soit disparu, soit fortement régressé. L'examen bibliographique révèle que ces espèces sont plus exposées au risque de perturbation du cycle sexuel que les espèces thermophiles. Cependant, les brèmes commune et bordelières qui les ont remplacées n'échappent pas non plus à l'évolution graduelle des traits : déclin de la longueur maximale, de la longévité, de la longueur et de l'âge de maturité mais aussi ajustement de l'occupation spatiale. Ainsi, la colonisation du domaine pélagique par les brèmes communes de l'année est devenue plus précoce lorsque le prérecrutement des gardons, perches et sandres s'est effondré.

L'ensemble de ces caractéristiques paraît découler des effets directs de la température qui contrôle plus ou moins directement la phénologie. Cependant la contamination cuprique perturbe l'environnement trophique avec une limitation potentielle des productions primaire et zooplanctoniques. Les poissons subissent donc des oscillations thermiques saisonnières avec des minima hivernaux élevés. Cette pression stabilisée dans sa gamme de variation saisonnière est probablement amplifiée par le réchauffement global (qu'il faudra apprécier) et par un facteur aggravant, la contamination cuprique en progression constante qui contribue à rendre la ressource trophique de plus en plus limitante, pesant à la fois sur la biomasse globale, la survie des poissons les plus grands et indirectement sur le renouvellement des générations.

L'enjeu des travaux à venir sera de préciser les traits biologiques notamment grâce à l'exploitation de la banque d'informations et du matériel biologique disponibles. Les études principalement descriptives ont permis d'établir les patrons de répartition spatiale au cours du développement ontogénétique du poisson (Flesch, 1994; Flesch et al., 1995; Marzou, 1996; Dembski, 2005). Ces études démographiques devront être complétées par une caractérisation approfondie de la structure des populations. L'identification des cohortes, des indices de condition, des stades de maturité confrontés aux conditions thermiques et trophiques doit permettre une approche intégrée s'appuyant sur des connaissances autoécologiques, 
biocénotiques et systémiques. La perche soleil fait l'objet d'une étude approfondie (examen histologique du cycle sexuel; Valente, 2008). Ces informations devront être renforcées par la comparaison des traits obtenus avec les caractéristiques des populations régionales. Malgré des caractéristiques physico-chimiques et trophiques différentes du Mirgenbach, le réservoir de Madine (bassin versant du Rupt de Mad, affluent senestre de la Moselle) pourrait constituer un témoin thermique, en raison de son faible éloignement géographique et de son appartenance aux plans d'eau artificiels de plaine calcaire. Dans le Mirgenbach, le domaine littoral doit faire l'objet d'une vigilance particulière. La réduction récente mais marquée des herbiers pourrait aggraver les conditions de reproduction du poisson, puis de développement de leur progéniture. Les organismes littoraux manifestent des relations trophiques complexes en raison de l'hétérogénéité de la ressource trophique et spatiale, du confinement de communautés différentes (végétaux macrophytes, périphyton, zooplancton, zoobenthos, ichthyofaune), de la succession d'espèces (jeunes stades ontogénétiques du poisson) et du rôle des espèces non indigènes (Dembski, 2005; Maazouzi, 2006; Maazouzi et al., 2007, 2008a, 2008b; Dembski et al., 2008). Dans le cas particulier de la perche soleil, ces relations trophiques seront prolongées par l'examen de contenus digestifs et de biomanipulations impliquant des représentants des réseaux trophiques littoraux. La disponibilité en proies potentielles et les conséquences directes (toxicologiques) et indirectes (trophiques) de la contamination cuprique pour la production secondaire devraient être recherchées. Deux articles ultérieurs concerneront l'évolution du planton et du macrozoobenthos sous l'influence conjointe de l'échauffement des eaux et de la présence de cuivre. Cependant, le déclin de la production primaire limitant la production secondaire, l'étude des relations trophiques devrait être approfondie dans les domaines littoraux et pélagiques afin d'examiner le rôle des différents composantes de la production primaire au sein des réseaux trophiques.

Depuis quelques années, les réservoirs sont l'objet d'un intérêt croissant en Europe, notamment en raison de la demande formulée par la Directive cadre européenne sur l'eau. Le réservoir du Mirgenbach appartient à la catégorie des plans d'eau artificiels de plaine calcaire. La nature des informations acquises doit interpeller les gestionnaires. Le diagnostic des masses d'eau stagnantes repose dans le cas de l'ichthyofaune, sur l'utilisation des filets maillants scandinaves (Appelberg et al., 1995; CEN \& AFNOR, 2005) dont la maille la plus élevée, $55 \mathrm{~mm}$ ne permet pas la capture des plus grands individus. Or l'évolution de leur longueur ou masse mériterait pourtant d'être suivie. La biométrie des captures a lieu in situ. La précision accordée à la pesée est satisfaisante pour estimer la biomasse collectée mais pourrait se révéler insuffisante pour apprécier les relations longueur masse puis caractériser la condition des populations et leur variation. L'âge individuel devrait également être déterminé dans un contexte où l'accroissement thermique accompagnant le changement global 
pourrait moduler la croissance individuelle et la structure démographique des populations.

\section{REMERCIEMENTS}

Cette synthèse a été menée dans le cadre du groupe Thermie et Hydrobiologie, conçu et animé par le Cemagref et Electricité de France, à la suite de l'épisode caniculaire en 2003. Les auteurs remercient la Direction recherche et développement (Électricité de France, Chatou) ainsi que le CNPE de Cattenom pour leur contribution financière lors de l'acquisition et de l'exploitation des données. Nous remercions Anne Pedon-Flesch et Nathalie Dubost ainsi que le personnel de leur cabinet pour leur collaboration. Ce travail n'aurait pu aboutir sans l'aide des très nombreuses personnes qui ont participé aux campagnes de pêche et au dépouillement des échantillons.

\section{RÉFÉRENCES BIBLIOGRAPHIQUES}

Andersson J., Sandström O. \& Hansen H.J.M., 1990. Elver (Anguilla anguilla L.) stockings in a Swedish thermal effluent - recaptures, growth and body condition. J. Appl. Ichthyol. 7 : 78-89.

Appelberg M., Berger H.M., Hesthagen T., Kleiven E., Kurkilahti M., Raitaniemi J. \& Rask M., 1995. Development and intercalibration of methods in Nordic freshwater fish monitoring. Water Air and Soil Pollution 85 : 401-406.

Baglinière J.L. \& Le Louarn H., 1987. Scales characteristics of the main fresh-water fish species in France. Bulletin Français de la Pêche et de la Pisciculture 306 : 2-39.
Balkuviené G. \& Pernaraviciuté B., 1994. Growth rates of roach (Rutilus rutilus (L.)) in a cooling water reservoir under different thermal conditions. Int. Revue ges. Hydrobiol. 79 (1) : 139-142.

Barbour C.D. \& Brown J.H., 1974. Fish species diversity in lakes. Am. Nat. 108 : 473-489.

Beitinger T.L., Bennett W.A. \& McCaulet R.W., 2000. Temperature tolerances of North American freshwater fishes exposed to dynamic changes in temperature. Environmental biology of fishes $58: 237-275$.

Bertonas E., 2002. Changes in fish biomass under impact of a thermal effluent and eutrophication in lake Druksiai. Acta Zoologica Lituanica 12 : 242-253.

Boytsov M.P., 1971. The effect of warm water discharged by the Konakova power point station on the distribution and growth of young fishes of Uven'kovo reservoir. J. Ichtyol. 11(2) : 257-262.

Brander K., 1996. Effects of climate change on cod (Gadus morhua) stocks. In Global warming : implications for freshwater and marine fish. Society for experimental biology. Seminar series 61 . Cambridge university press. Wood C.M. \& McDonald D.G. (Eds.) : 255-278.

Bruslé J. \& Quignard J.-P., 2004. Les poissons et leur environnement. Ecophysiologie et comportements adaptatifs. Paris : Éditions Tec \& Doc. Lavoisier.

Burroughs W.J., 2001. Climate change. A multidisciplinary approach. Cambridge University press.

CEN \& AFNOR, 2004. Détermination de l'indice poisson rivière (IPR). NF T90$344: 16 p$.

CEN \& AFNOR, 2005. Water quality Sampling of fish with multi-mesh gillnets. NF EN 14757. 26p.

Copp G.H., Fox M.G., Przybylski M., Godinho F.N. \& Vila-Gispert A., 2004. 
Life-time growth patterns of pumpkinseed Lepomis gibbosus introduced to Europe, relative to native North American populations. Folia Zoologica 53 : 237-254.

Daufresne M., Roger M.C., Capra H. \& Lamouroux N., 2003. Long-term changes within the invertebrate and fish communities of the upper Rhône river: effects of climatic factors. Global Change Biology 10 : 124-140.

Degiorgi F. \& Grandmottet J.-P., 1993. Spatial relations between lacustrine ichthyofauna and a predefined partition of the lacustrine basin. Bulletin Francais de la Pêche et de la Pisciculture 66(329) : 199-220.

Degiorgi F., Grandmottet J.-P., Raymond J.-C. \& Rivier B., 2001. Échantillonnage de l'ichtyofaune lacustre : engins passifs et protocole de prospection. Exemple des filets maillants et emmêlants. In Gestion piscicole des grands plans d'eau. INRA. Gerdeaux D. (Ed.) : 151-181.

Dembski S., 2005. Stratégies d'occupation spatiale en milieu lacustre : réponses de l'ichtyofaune dans un réservoir échauffé, non stratifié. Thèse de doctorat: Sciences de la Vie, Hydrobiologie. Université de Metz (école doctorale RP2E).

Dembski S., Vinot I., Masson G., Pihan J.C., 2005. Gestion et différents usages de la retenue du Mirgenbach associée à la centrale électronucléaire de Cattenom. P2. Ponts et Chaussées. 8p.

Dembski S., Masson G., Monnier D., Wagner P. \& Pihan J.-C., 2006. Consequences of elevated temperatures on life-history traits of an introduced fish, pumpkinseed Lepomis gibbosus. Journal of Fish Biology 68 : 1-16.

Dembski S., Masson G. \& Pihan J.-C., 2007. État écologique de la retenue du Mirgenbach (Cattenom, France) :
Synthèse de données des compartiments biologiques (1988-2005). Rapport contrat Laboratoire IEBE, Université Paul Verlaine, Metz/EDF-R\&D.

Dembski S., Masson G., Wagner P. \& Pihan J.-C., 2008. Habitat use by YOY in the littoral zone of an artificially heated reservoir. International Review of Hydrobiology 93 : 243-255.

Downing J.A., Plante C. \& Labonde S., 1990. Fish production correlated with primary productivity, not the morphoedaphic index. Canadian Journal of Fischeries and Aquatic Sciences 47 : 1929-1936.

Dubost N., 1996. Voies de diversification de l'élevage de la carpe Cyprinus carpio L. en Lorraine : avantages, contraintes. Thèse de doctorat: Sciences de la Vie, Hydrobiologie. Université de Metz.

Flesch A., 1994. Biologie de la perche (Perca fluviatilis) dans le réservoir du Mirgenbach (Cattenom, Moselle). Thèse de doctorat: Sciences de la Vie, Hydrobiologie. Université de Metz.

Flesch A., Masson G. \& Moreteau J.-C., 1994. Comparaison de trois méthodes d'échantillonnage utilisées dans l'étude de la répartition de la perche (Perca fluviatilis) dans un lac-réservoir. Cybium 18 : 39-56.

Flesch A., Masson G. \& Moreteau J.-C., 1995. Temporal distribution of perch (Perca fluviatilis L.) in a lake-reservoir (Moselle, France) : analysis of catches with vertical gill nets. Hydrobiologia 300/3001 : 335-343.

Gajdusek J., Astrauska A., Virbickas J., Lucksiene D. \& Misuniene D., 1987. The ecology of bream in the cooling reservoir of a Lithuanian Thermal power station, Acta scientiarum naturali Academiae scientiarum bohemoslovacae- Brno 21(6) : 1-36.

George G., Hurley M. \& Hewitt D., 2007. The impact of climate change on the physical characteristics of the larger 
lakes in the English Lake District. Freshwater Biology 52 : 1647-1666.

Gillet C. \& Quétin P., 2006. Effect of temperature changes on the reproductive cycle of roach in lake Geneva from 1983 to 2001. Journal of fish biology $69: 518-534$.

Glantz M.H., 1992. Climate variability, climate change and fisheries. Cambridge university press.

Gosse P., 2008. Caractérisation du phosphore, de l'azote et de la chlorophylle a dans l'eau autour de la centrale de Cattenom (1992 - 1995) : Moselle, lac de Mirgenbach, prise et rejet d'eau du site, Rapport EDF H-P76-200701308-FR.

Irz P., Argillier C. \& Oberdorff T., 2004a. Native and introduced fish species richness in French lakes : local and regional influences. Global Ecology and Biogeography 13 : 335-344.

Irz P., Argillier C. \& Proteau J.-P., 2004b. Contribution of native and non-native species to fish communities in French reservoirs. Fisheries Management and Ecology 11 : 165-172.

Jobling M., 1994. Fish bioenergetics. London : Chapman \& Hall.

Jobling M., 1997. Temperature and growth : modulation of growth rate via temperature change. In Global warming : implications for freshwater and marine fish. Society for experimental biology. Seminar series 61. Cambridge university press. Wood C.M. \& McDonald D.G. (Ed.) : 225-253.

Karas P. \& Neuman E., 1981. First-year growth of perch (Perca fluviatilis) and roach (Rutilus rutilus) in heated Baltic bay. Reports of the Institute of Freshwater Research 59 : 48-63.

Keith P. \& Allardi J.E., 2001. Atlas des poissons d'eau douce de France. Patrimoines naturels, 47. Paris : M.N.H.N.

Krovin A.S. \& Rodionov S.N., 1992. Atlanto-Scandian herring: a case study. In Climate variability, climate change and fisheries. Cambridge university press. Glantz M.H. (Ed.) : 231-260.

Law R., 2001. Phenotypic and genetic changes due to selective exploitation. In Conservation of exploited species. Conservation biology 6. Cambridge university press. Reynolds J.D., Mace G.M., K.H. Redford \& Robinson J.G. (Eds.) : 323-342.

Luksiene D. \& Sandstrom O., 1994. Reproductive disturbance in a roach (Rutilus rutilus) population effected by cooling water discharge. Journal of fish biology 45 : 613-625.

Luksiene D., Sandstrom O., Lounasheimo L. \& Andersson J., 2000. The effects of thermal effluent exposure on the gametogenesis of female fish. Journal of Fish Biology 56 : 37-50.

Maazouzi C., 2006. Utilisation des acides gras comme marqueurs de la matière organique dans la chaîne trophique d'une retenue artificielle (Mirgenbach Nord-Est France). Thèse de Doctorat Sciences de la vie «label européen », Université Paul Verlaine - Metz (école doctorale RP2E).

Maazouzi C., Masson G., Izquierdo M.S. \& Pihan J.-C., 2007. Fatty acid composition of the amphipod Dikerogammarus villosus : Feeding strategies and trophic links. Comp Biochem Physiol $A$ 147(4) : 868-875.

Maazouzi C., Masson G., Izquierdo M.S. \& Pihan J.-C., 2008a. Midsummer heat wave effects on lacustrine plankton: variation of assemblage structure and fatty acid composition. Journal of thermal Biology 33 : 287-296.

Maazouzi C., Masson G., Izquierdo M.S. \& Pihan J.-C., 2008b. Chronic copper exposure and fatty acid composition of the amphipod Dikerogammarus villosus : results from a field study. Environmental pollution 156 : 221-226.

Marzou R., 1996. Étude des crustacés planctoniques d'une retenue soumise à des rejets thermiques : description 
dynamique et relations avec le peuplement pisciaire. Thèse, Doctorat en sciences de la vie. Hydrobiologie, Université de Metz.

Masson G., Pedon-Flesch A. \& Marzou R., 2001. Méthodes de pêche active en milieu lacustre : caractéristiques et contraintes d'utilisation. In, Gestion piscicole des grands plans d'eau. INRA. Gerdeaux D. (Ed.) : 109149.

Mattheeuws A., Genin M., Detollenaere A. \& Micha C., 1981. Étude de la reproduction du gardon (Rutilus rutilus) et des effets d'une élévation provoquée de la température en Meuse sur cette reproduction. Hydrobiologia 85 : 271-282.

McCarthy I.D. \& Houlihan D.F., 1997. The effect of temperature on protein metabolism in fish : the possible consequences for wild Atlantic salmon (Salmo salar L.) stocks in Europe as a result of global warming. In Global warming : implications for freshwater and marine fish. Society for experimental biology. Seminar series 61. Cambridge university press. Wood C.M. \& McDonald D.G. (Eds.) : 51-77.

Mehner T., Holmgren K., Lauridsen T.L., Jeppesen E. \& Diekmann M., 2007. Lake depth and geographical position modify lake fish assemblages of the European 'Central Plains' ecoregion. Freshwater biology 52 : 2285-2297.

Mersch J., Dubost N. \& Pihan J.-C., 1993. Comparaison de plusieurs supports analytiques inertes et biologiques pour apprécier la contamination de la retenue du site électronucléaire de Cattenom, France. Annls Limnol. 29 : 325337.

Oberdorff T., Pont D., Hugueny B. \& Porchers J.-P., 2002. Development and validation of a fish-based index for the assessment of 'river health' in France. Freshwater Biology 47 : 1720-1734.
Panfili J., de Pontual H., Troadec H. \& Wright P.J., 2002. Manuel de sclérochronologie des poissons. Coédition Ifremer-IRD.

Pauly D. \& Moreau J., 1997. Méthodes pour l'évaluation des ressources halieutiques. Collection Polytech de I'INP de Toulouse. Cépaduès-éditions. Toulouse.

Persson L., Diehl S., Johansson L., Andrersson G. \& Hamrin S.F., 1991. Shifts in fish communities along the productivity gradient of temperate lakes-patterns and the importance of size-structured interactions. Journal of Fish Biology 38 : 281-293.

Pertoldi C. \& Bach L.A., 2007. Evolutionary aspects of climate-induced changes and the need for multidisciplinarity. $J$. Therm. Biol. 32 : 118-124.

Pihan J.-C. (Coord.), 1986 à 2005. Étude hydrobiologique de la retenue du Mirgenbach. Contrat EDF REAL et EDF CNPE Cattenom, Université de Metz. Rapports annuels.

Pinder A.C., 2001. Keys to larval and juvenile stages of coarse fishes from fresh waters in the British Isles. Freshwater biological association. Scientific publication $\mathrm{n}^{\circ} 60$.

Poirel A., Merle G., Salençon M.-J. \& Travade F., 2001. Gestion hydraulique et ressources piscicoles dans les retenues hydro-électriques. In Gestion piscicole des lacs et retenues artificielles. Paris : INRA. Gerdeaux D. (Ed.) : 3-34.

Pronier O., 2000. Analyse des peuplements ichthyologiques des plans d'eau français et perspectives de gestion piscicole. Thèse de doctorat de l'INPTENSAT. Spécialité sciences agronomiques.

Reynolds J.D., Jennings S. \& Dulvy N.K., 2001. Life histories of fishes and population responses to exploitation. In Conservation of exploited species. Conservation biology 6. Cambridge university press. Reynolds J.D., Mace 
G.M., Redford K.H. \& Robinson J.G. (Eds.) : 148-168.

Rinchard J. \& Kestemont P., 1996. Comparative study of reproductive biology in single and multiple-spawner cyprinid fish. I. Morphological and histological features. Journal of fish biology 49 : 883-894.

Spillmann C.J., 1961. Poissons d'eau douce, P. Lechevallier (Faune de France ; 65), Paris.

Thorpe J.E., 1977. Morphology, physiology, behaviour and ecology of Perca fluviatilis L. and Perca flavescens Mitchill. Journal of the Fisheries Research Board of Canada 34 : 1504-1514.

Université de Metz, UER écologie \& Institut européen d'écologie, 1977. Constat hydrobiologique prélimiaire du secteur intéressé par le projet d'implantation d'une centrale nucléaire sur le site de Cattenom. Convention EDF, 1976.

Urho L., 1996. Identification of perch (Perca fluviatilis), pikeperch (Stizostedion lucioperca) and ruffe (Gymnocephalus cernuus) larvae. Ann. Zool Fennici 33 (3-4) : 659-667.

Valente E., 2008. Traits biologiques d'une espèce invasive, la perche soleil ( $\mathrm{Le}$ pomis gibbosus), dans un réservoir artificiel aux eaux échauffées : reproduction, croissance, longévité. Thèse de Doctorat Sciences de la vie, Université Paul Verlaine - Metz (école doctorale RP2E).

Van der Kraak G. \& Pankhurst N.W., 1997. Temperature effects on the reproductive performance of fish. In Global warming : implications for freshwater and marine fish. Society for experimental biology. Seminar series 61. Cambridge university press. Wood C.M. \& McDonald D.G. (Eds.) : 159-176.

Vein D., Gigleux M., Flesch A., Pierre J.-F., Marzou R. \& Pihan J.-C., 1990. Trophic evolution of a reservoir with overheated waters : nuclear power station at Cattenom, Moselle, East France. Annales de Limnologie - International Journal of Limnology 27 : 87-98.

Verneaux J., 1968. Le milieu et les peuplements aquatiques - Relations. B.T.I. 227 : 245-260.

Villeneuve F., Copp G.H., Fox M.G. \& Stakènas S., 2005. Interpopulation variation in growth and life-history traits of the introduced sunfish, pumpkinseed Lepomis gibbosus, in southern England. J. Appl. Ichthyol. 21 : 275-281.

Vinot I., 2004. Impact des rejets de cuivre et zinc en milieu aquatique : circulation, spéciation, biodisponibilité, bioaccumulation, transferts et toxicité. Thèse de doctorat. Sciences de la vie : Université de Metz (école doctorale RP2E).

Vinot I. \& Pihan J.-C., 2005. Circulation of copper in the biotic compartments of a freshwater damned reservoir. Environmental Pollution 133 : 169-182.

Winfield I.J., George D.G., Fletcher J.M. \& Hewitt D.P., 1998. Environmental factors influencing the recruitment and growth of underyearling perch (Perca fluviatilis) in Windermere North Basin, UK, from 1966 to 1990. In Workshop on management of lakes and reservoirs during global change. Proceedings of NATO Advanced research. Kluwer Academic Publishers, Dordrecht. George D.G., Jones J.G., Puncochar P., Reynolds C.S. \& Sutcliffe D.W. (Eds.) : 245-261.

Winner R.W. \& Owen H.A., 1991. Toxicity of copper to Chlamydomonas reinhardtii (Chlorophyceae) and Ceriodaphnia dubia (Crustacea) in relation to changes in water chemistry of a freshwater pond. Aquatic Toxicology $21: 157-170$.

Wood C.M. \& McDonald D.G., 1997. Global warming : implications for freshwater and marine fish. Society for experimental biology. Seminar series 61 . Cambridge university press. 
